\title{
Review Article \\ Electrosynthesis and Spectroscopic Characterization of Poly(o-Aminophenol) Film Electrodes
}

\author{
Ricardo Tucceri, ${ }^{1}$ Pablo Arnal, ${ }^{2}$ and Alberto Scian ${ }^{2}$ \\ ${ }^{1}$ Instituto de Investigaciones Fisicoquímicas Teóricas y Aplicadas (INIFTA), Facultad de Ciencias Exactas, \\ Universidad Nacional de La Plata, Sucursal 4, Casilla de Correo 16, 1900 La Plata, Argentina \\ ${ }^{2}$ Centro de Tecnología de Recursos Minerales y Cerámica (CETMIC), CIC-CONICET La Plata, C.C. 49, B1897ZCA, \\ B. Gonnet-Pcia, Buenos Aires, Argentina \\ Correspondence should be addressed to Ricardo Tucceri, rtucce@gmail.com
}

Received 19 January 2012; Accepted 7 February 2012

Academic Editors: M. Karakisla, M. Sanopoulou, and B. G. Soares

Copyright ( $) 2012$ Ricardo Tucceri et al. This is an open access article distributed under the Creative Commons Attribution License, which permits unrestricted use, distribution, and reproduction in any medium, provided the original work is properly cited.

\begin{abstract}
This review, which is divided into three parts, concerns electrochemical synthesis, spectroscopic characterization, and formation mechanisms of poly(o-aminophenol) (POAP) film electrodes. The first part of this review is devoted to describe the electropolymerization process of $o$-aminophenol on different electrode materials and in different electrolyte media by employing both potentiodynamic and potentiostatic methods. The second part refers to spectroscopic studies carried out by different authors to both, identify the products of the $o$-aminophenol electrooxidation and elucidate the chemical structure of poly $(o$-aminophenol $)$ film electrodes. The third part shows the different mechanisms formulated to interpret the POAP films formation from both acid and basic solutions of $o$-AP. Also, some electrochemical and spectroscopic data which allowed to propos the corresponding formation mechanisms, especially in basic media, are described.
\end{abstract}

\section{Introduction}

Oxidation of $o$-aminophenol on different electrode materials (gold, platinum, carbon, indium-tin oxide, etc.) in aqueous medium was shown to form poly-o-aminophenol (POAP). Like aniline, $o$-AP can be polymerized electrochemically in acidic, neutral, and alkaline solutions. However, while a conducting film is only formed in acidic media, POAP synthesized in neutral and alkaline media leads to a nonconducting film.

Electropolymerization of $o$-AP in acid medium yields an electroactive polymer that exhibits its maximal electroactivity within the potential range $-0.2 \mathrm{~V}<E<0.5 \mathrm{~V}$ (versus SCE) at $\mathrm{pH}$ values lower than $3[1,2]$. The electroactivity of POAP was explained by a redox mechanism that involves an addition/elimination of protons coupled with a reversible electron transfer [2]. The charge-transport process at POAP films was studied by employing different electrochemical techniques [1-7]. In previous work [8], we studied the electrochemistry of POAP in the presence of different active redox couples in solution, and it was demonstrated that external variables, such as film thickness, solution $\mathrm{pH}$ and redox-couple concentration in solution, affect both the permeation process of electroactive species and the electron motion through a POAP film. An interesting review concerning charge-transfer and charge-transport processes occurring in the curse of redox reactions of POAP film electrodes can be found in [9].

The properties of POAP synthesized in basic medium are favorable to its applications in the field of bioelectrochemistry and electrocatalysis. In this regard, a nonconducting material is obtained that exhibits several advantages with respect to other polymers. POAP can be synthesized on different electrode surfaces, and the polymer thickness can be controlled within $10-100 \mathrm{~nm}$ due to a self-limiting growth. Employed as material to build amperometric biosensors, POAP has shown to be permselective. In this regard, the interference from electroactive species, such as ascorbic acid and uric acid, which are generally encountered in the determination of physiological sample, was significantly reduced 
by the use of a POAP film. Also, POAP is able to immobilize biological macromolecules. It was proved that large amounts of glucose oxidase could be immobilized at POAP films, which is essential to the good performance of an amperometric glucose sensor. In the field of electrocatalysis, an interesting characteristic of POAP is the presence of an electron-donating $\mathrm{OH}$ group next to the imine nitrogen that increases the electron density at the imine sites. In addition, $\mathrm{OH}$ by itself is also a potential coordinating site. These factors combine to provide a strong acceptor binding of POAP with several metal cations. In this regard, POAP was employed to develop stable electrocatalysts for oxygen reduction. A review about practical applications of POAP is found in [11].

Despite the 2 reviews $[9,11]$ reported in the literature about charge conduction and practical applications of POAP film electrodes, respectively, according to our knowledge, any review work has been reported until now about electrosynthesis and spectroscopic characterization of poly $(o-$ aminophenol) films from both neutral and alkaline solutions of $o$-aminophenol. It is expected that the present review, together with the other two previously published $[9,11]$, will be helpful to the colleagues who pretend to achieve a more complete knowledge about the particular properties of this polymer.

\section{Electropolymerization of o-Aminophenol on Different Electrode Materials and in Different Electrolyte Media: Formation of Poly(o-Aminophenol) Film Electrodes}

Table 1 shows electrode materials, electrosynthesis methods, and electrolyte composition employed by different authors to obtain POAP films.

\subsection{Electropolymerization of o-Aminophenol in Acidic Media.} Barbero et al. [10] reported a study about oxidation of $o$-AP and closely related compounds employing electrochemical, chemical, and spectroscopic measurements. The electrooxidation of $o$-AP was studied on different electrode materials (Pt, Au, and glassy carbon) and different electrolyte media $(1<\mathrm{pH}<7)$. A typical voltammogram of a $\mathrm{Pt}$ electrode contacting a $0.1 \mathrm{M} \mathrm{HClO}_{4}+0.4 \mathrm{M} \mathrm{NaClO}_{4}+1 \times 10^{-3} \mathrm{M}$ $o$-AP aqueous solution ( $\mathrm{pH} 1)$ is shown in Figure 1 .

On the first positive sweep, two peaks are defined, a, at $0.60 \mathrm{~V}$ (SCE) attributed to the oxidation of $o$-AP to monocation radical $\left(o-\mathrm{AP}^{\bullet+}\right)$ and another peak, b, at $0.85 \mathrm{~V}$, which was assigned to the oxidation of $\left(o-\mathrm{AP}^{\bullet+}\right)$ to dication. On the negative sweep, none of these peaks show complementary peaks, indicating chemical followup reactions giving products detected as peaks $\mathrm{c}-\mathrm{c}^{\prime}$ and $\mathrm{d}-\mathrm{d}^{\prime}$ on the subsequent sweeps. It was observed that the system $c-c^{\prime}$ diminishes after continuous cycling in the same way as a, but the peak system $\mathrm{d}-\mathrm{d}^{\prime}$ increases, besides showing the characteristic behavior of a deposited electroactive substance. This was verified by stirring the solution while cycling, because the system $\mathrm{d}-\mathrm{d}^{\prime}$ remained unchanged, as expected for an irreversible adsorbed electroactive substance. Analysis of the products employing IR and UV-V is spectroscopy (see below) showed that

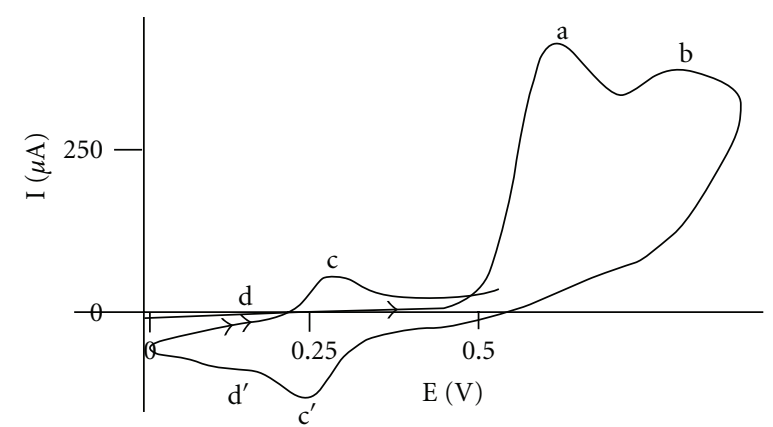

Figure 1: Cyclic voltammogram of a Pt electrode contacting a $0.1 \mathrm{M}$ $\mathrm{HClO}_{4}+0.4 \mathrm{M} \mathrm{NaClO}_{4}+1 \times 10^{-3} \mathrm{M}$ o-AP solution, $\mathrm{pH} 1$. Scan rate, $v=0.1 \mathrm{~V} \mathrm{~s}^{-1}$, electrode area $A=0.126 \mathrm{~cm}^{2}$ [10].

the couple $\mathrm{d}-\mathrm{d}^{\prime}$ corresponds to a polymer of 3-aminophenoxazone (3APZ). A mechanism for the $o$-AP electrooxidation was proposed in [10] where the $\left(o-\mathrm{AP}^{\bullet+}\right)$, formed in the first charge transfer step, may follow different reaction paths (see below). Thus, the formation of a composite of two different films, one of linear chain structure similar to polyaniline (PANI) and the other with a phenoxazine-like chain structure, was proposed in [10]. The latter was considered to be the predominant product. In order to reinforce the conclusion about the proposed structure for the electroactive substance, the electrochemical properties of several compounds ( $o$-anisidine, $p$ - and $m$-aminophenol, $o$-phenylenediamine, etc.) with similar structures to $o$-AP were also analyzed in [10]. The presence of phenoxazine units in the structure of the polymer formed by oxidation of $o$-AP was also confirmed by Salavagione and coworkers in another work [24] by employing FTIR spectroscopy (see Section 2). The electroactive substance formed by oxidation of $o$-AP was denominated poly-o-aminophenol (POAP). An analysis of the voltammetric peak current $\left(i_{\mathrm{pa}}\right)$ as a function of the sweep rate $(\nu)$ and the initial concentration of $o$-AP was also carried out in [10]. It was observed that high concentrations of monomer favor higher rates of polymerization, with the consequent partial blocking of the electrode surface. This effect was more noticeable at high temperatures. However, the blocking effect was negligible at low monomer concentrations, and then the normal behavior for the cation radical dimerization reactions was observed. Barbero et al. [10] also prepared the electroactive polymer by chemical oxidation of $o$-AP and its properties were compared with those of the electrochemically produced POAP. The chemical synthesis of POAP confirmed that the actual monomer in the formation of the polymer is the cyclic dimer of $o$-AP, 3APZ. A calculation of a global value of the rate constant for the dimerization reaction was carried out in [10] (see Section 3 ). With regard to the solution $\mathrm{pH}$, similar voltammetric response as shown in Figure 1 was observed for $\mathrm{pH}$ values lower than 3. At $\mathrm{pH}$ values between 3 and 7 , a diminishing in the current of the peak system $\mathrm{d}-\mathrm{d}^{\prime}$, assigned to the film formation, was observed. Barbero et al. [10] remark that if extreme care is not taken in the preparation of a POAP film, not only in the concentration but also in the potential ranges, the possibility of side reactions and 
TABLE 1: Electropolymerization of $o$-aminophenol on different electrode materials and in different electrolyte media.

\begin{tabular}{|c|c|c|c|}
\hline Electrode materials & Electrolyte medium & Electrosynthesis method & Reference \\
\hline $\mathrm{Pt}, \mathrm{Au}$, and glassy carbon & $\begin{array}{l}0.1 \mathrm{M} \mathrm{HClO}_{4}+0.4 \mathrm{M} \mathrm{NaClO}_{4}+ \\
1 \times 10^{-3} \mathrm{M} o-\mathrm{AP}(1<\mathrm{pH}<7)\end{array}$ & $\begin{array}{l}\text { Potentiodynamic cycling - } \\
0.25 \mathrm{~V} \text { and } 0.70 \mathrm{~V} \text { (SCE) }\end{array}$ & {$[10]$} \\
\hline Pt electrode & $\begin{array}{l}0.4 \mathrm{~mol} \mathrm{~cm}^{-3} \text { of } \mathrm{NaClO}_{4}+ \\
10^{-2} \mathrm{~mol} \mathrm{dm}^{-3} \text { of } o-\mathrm{AP}\end{array}$ & $\begin{array}{l}\text { Potentiodynamic cycling }-0.25 \mathrm{~V} \\
\text { and } 0.75 \mathrm{~V} \text { (SCE) }\end{array}$ & {$[12]$} \\
\hline Carbon paste electrode & $\begin{array}{l}5 \mathrm{mM} \text { o-AP+ } 0.5 \mathrm{M} \mathrm{HClO}_{4} \text { in the } \\
\text { presence and in the absence of } \\
\text { sodium dodecyl sulfate }\end{array}$ & $\begin{array}{l}\text { Potentiodynamic cycling }-0.1 \mathrm{~V} \\
\text { and } 0.7 \mathrm{~V}(\mathrm{Ag} / \mathrm{AgCl} / \mathrm{KCl} 3 \mathrm{M})\end{array}$ & {$[13]$} \\
\hline $\begin{array}{l}\text { Platinum and glassy carbon } \\
\text { electrodes }\end{array}$ & $\begin{array}{l}0.2 \mathrm{M} \mathrm{NaClO}_{4}+0.1 \mathrm{M} \mathrm{HClO}_{4}+ \\
5 \times 10^{-3} \mathrm{M} o \text {-AP solution }\end{array}$ & $\begin{array}{l}\text { Potentiodynamic cycling } 0.2 \mathrm{~V} \\
\text { and } 1.3 \mathrm{~V} \text { (RHE) }\end{array}$ & {$[14]$} \\
\hline $\begin{array}{l}\text { Glassy carbon electrodes } \\
\text { chemical (nitric acid ( } 67 \% \\
\mathrm{wt} / \mathrm{wt}) \text { and sulfuric acid } \\
(98 \% \mathrm{wt} / \mathrm{wt}) \text { for } 10 \mathrm{~min}) \\
\text { and electrochemically } \\
(1.85 \mathrm{~V} \text { (SCE) for } 5 \mathrm{~min}) \\
\text { pretreated before } \\
\text { electropolymerization of } \\
o-\mathrm{AP}\end{array}$ & $0.1 \mathrm{M} \mathrm{H}_{2} \mathrm{SO}_{4}+0.05 \mathrm{M} o-\mathrm{AP}$ & $\begin{array}{l}\text { Potentiodynamic method } \\
(-0.2 \mathrm{~V} \text { and } 0.7 \mathrm{~V} \text { versus SCE })\end{array}$ & {$[15]$} \\
\hline $\begin{array}{l}\text { Basal-plane pyrolytic } \\
\text { graphite and In-Sn oxide } \\
\text { conducting glass }\end{array}$ & $\begin{array}{l}0.5 \mathrm{M} \mathrm{Na}_{2} \mathrm{SO}_{4} \text { solution }(\mathrm{pH} 1)+ \\
50 \mathrm{mM} o \text {-AP }\end{array}$ & $\begin{array}{l}\text { Potentiodynamic cycling }(-0.4 \\
\text { and } 1.0 \mathrm{~V} \text { versus sodium chloride } \\
\text { saturated calomel electrode })\end{array}$ & {$[16]$} \\
\hline Glassy carbon & $\begin{array}{l}1 \mathrm{M} \mathrm{SO}_{4} \mathrm{H}_{2}+0.5 \mathrm{M} \mathrm{Na}_{2} \mathrm{SO}_{4}+ \\
50 \mathrm{mM} o \text {-AP solution }\end{array}$ & $\begin{array}{l}\text { Potentiodynamic cycling }(-0.2 \mathrm{~V} \\
\text { and } 1.0 \mathrm{~V} \text { versus SCE) }\end{array}$ & {$[17]$} \\
\hline $\begin{array}{l}\text { Glassy carbon and Pt } \\
\text { electrodes }\end{array}$ & $\begin{array}{l}0.05 \mathrm{M} o-\mathrm{AP} \text { in a mixture of } 1 \mathrm{M} \\
\mathrm{H}_{2} \mathrm{SO}_{4} \text { and } 0.5 \mathrm{M} \mathrm{Na}_{2} \mathrm{SO}_{4}\end{array}$ & $\begin{array}{l}\text { Potentiodynamic cycling }(-0.2 \mathrm{~V} \\
\text { to } 0.8 \mathrm{~V} \text { versus SCE) }\end{array}$ & {$[18]$} \\
\hline $\mathrm{Pt}$ and $\mathrm{Au}$ electrodes & $\begin{array}{l}0.05 \mathrm{M} o-\mathrm{AP}+0.5 \mathrm{M} \\
\mathrm{H}_{2} \mathrm{SO}_{4} \text { solution }\end{array}$ & $\begin{array}{l}\text { Potentiostatic method }(E=0.7 \\
\text { V; } 0.8 \mathrm{~V} \text { and } 0.9 \mathrm{~V} \text { versus SCE }\end{array}$ & {$[19]$} \\
\hline Pt and GC electrodes & $\begin{array}{l}0.05 \mathrm{M} \mathrm{o} \text {-aminophenol solution } \\
\text { in } 0.5 \mathrm{M} \mathrm{HClO}_{4}+10 \mathrm{mM} \\
\text { sulfonated nickel phtalocyanine }\end{array}$ & $\begin{array}{l}\text { Potentiodynamic cycling }(-0.25 \\
\text { and } 0.7 \mathrm{~V} \text { versus SCE) }\end{array}$ & {$[20]$} \\
\hline GC electrodes & $0.10 \mathrm{M} \mathrm{HClO}_{4}+0.10 \mathrm{M} \mathrm{o-AP}$ & $\begin{array}{l}\text { Potentiodynamic }(-0.10 \mathrm{~V} \text { and } \\
1.00 \mathrm{~V} \text { versus } \mathrm{SCE}) \text { and } \\
\text { potentiostatic }(E=1.0 \mathrm{~V} \text { for a } \\
\text { given time) methods }\end{array}$ & {$[21]$} \\
\hline $\begin{array}{l}\text { Vitreous carbon, platinum, } \\
\text { and copper }\end{array}$ & $\begin{array}{l}0.3 \mathrm{M} \mathrm{NaOH} \text { hydroalcoholic } \\
\text { solution }\left(70 \text { vol } \% \mathrm{H}_{2} \mathrm{O}, 30 \text { vol\% }\right. \\
\left.\mathrm{CH}_{3} \mathrm{OH}\right)+0.1 \mathrm{M} \text { o-AP }\end{array}$ & Potentiodynamic cycling & {$[22]$} \\
\hline $\begin{array}{l}\text { Glassy carbon electrode or } \\
\text { on a glass plate covered } \\
\text { with semiconducting } \\
\text { indium thin oxide }\end{array}$ & $\begin{array}{l}1 \mathrm{M} \text { phosphate buffer } \mathrm{pH} 5.55 \\
\text { solution containing } 25 \mu \mathrm{g} / \mathrm{mL} \text { of } \\
\text { laccase and } 10 \mathrm{mM} o-\mathrm{AP}\end{array}$ & $\begin{array}{l}\text { Potentiodynamic cycling }(-0.1 \mathrm{~V} \\
\text { and } 0.9 \mathrm{~V} \text { versus } \mathrm{Ag} / \mathrm{AgCl})\end{array}$ & {$[23]$} \\
\hline
\end{tabular}

consequently "side" polymers increases, and the real structures of the films obtained could be quite complex. Then, the best conditions proposed in [10] for obtaining a reproducible POAP film are the repetitive cycling between $-0.25 \mathrm{~V}$ and $0.70 \mathrm{~V}$ (SCE) of an $o$-AP aqueous acid solution ( $\mathrm{pH} 1$ ) and an $o$-AP concentration less than $20 \mathrm{mM}$. Figure 2 shows the voltammetric response of POAP in a monomer-free solution. The voltammogram is highly asymmetric, suggesting a complex redox behavior that does not follow the simple Nernstian model. The phenoxazine-like chain structure proposed by Barbero et al. [10] is shown in Figure 3, which shows the redox switching of POAP in acidic media.
The electrochemical formation of POAP was also described by Ortega in [12]. Ortega focused his attention on the monomer purification before electropolymerisation. 0 -AP (purum 90\%) was purified by recrystallizing it three times in ethyl acetate. The very pale white plates were dried in a warm water bath under vacuum to eliminate residual solvent. The monomer was stored in a desiccator under vacuum until required. $\mathrm{MNR}, \mathrm{IR}$, and $\mathrm{C}^{13}$ spectra were recorded in order to ensure the absence of contaminant oxidation species in the monomer. A typical cyclic multisweep voltammogram of the Pt electrode in contact with a $0.4 \mathrm{~mol} \mathrm{~cm}^{-3}$ of $\mathrm{NaClO}_{4}$ and $10^{-2} \mathrm{~mol} \mathrm{dm}^{-3}$ of monomer solution is shown in Figure 4. 


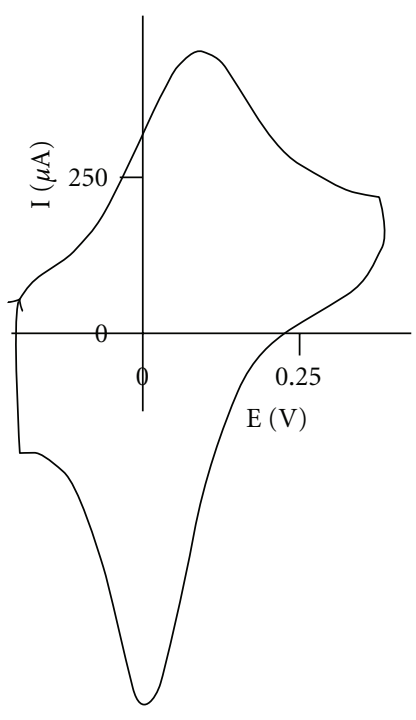

FIGURe 2: Cyclic voltammogram of POAP at $\mathrm{pH}=1$, scan rate, $v=$ $0.1 \mathrm{~V} \mathrm{~s}^{-1}$, Pt electrode, electrode area $A=0.126 \mathrm{~cm}^{2}$, supporting electrolyte: $0.4 \mathrm{M} \mathrm{NaClO}_{4}+0.1 \mathrm{M} \mathrm{HClO}_{4}[10]$.

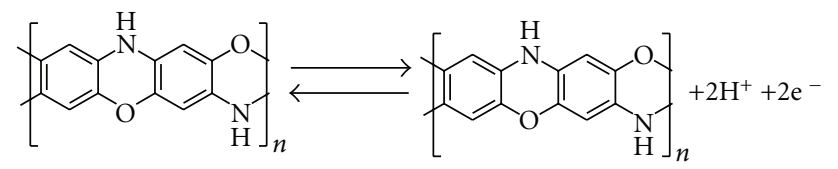

FIGURE 3: Reaction scheme of POAP redox switching including oxidized and reduced forms [10].

The electrode potential was continuously swept at $100 \mathrm{mV} \mathrm{s}^{-1}$ between -0.25 and $0.75 \mathrm{~V}$ (SCE) in [12]. On the first positive seep, only one irreversible peak is defined at $0.65 \mathrm{~V}$ (peak a, only visible at lower sensibility) which was attributed to the oxidation of the monomer to the monocation radical. No complementary peak to a was observed on the negative scan, while a large peak appears at $0.23 \mathrm{~V}$ (peak b). This peak has a complementary one (peak e) at $0.29 \mathrm{~V}$ in the next positive scan, and it was assigned to the redox reaction of a dimer formed prior to cyclisation to produce $3 \mathrm{APZ}$. It was also observed that while peak a rapidly and continuously decreases up to a minimum, peaks b and e fall correspondingly. Also, after 8 or 10 min cycling, the current response in the potential range from 0.0 to $0.2 \mathrm{~V}$ begins to increase continuously with successive potential scans, giving rise to peaks $\mathrm{c}$ and $\mathrm{d}$, which were assigned to the buildup of an electroactive polymeric product on the electrode. Neither polymer nor couple b and e were formed if the potential scan did not reach $0.65 \mathrm{~V}$. On the other hand, peaks from $\mathrm{b}$ to $\mathrm{d}$ were not observed when potential cycling takes place under continuous nitrogen bubbling. Thus, Ortega indicates that a slow chemical reaction is associated to the peaks $\mathrm{b}$ and $\mathrm{e}$ and under stirred conditions the intermediate corresponding to $\mathrm{b}$ and e peaks is moved away from the electrode, so that its further transformation into polymer is prevented. The comparison of voltammograms

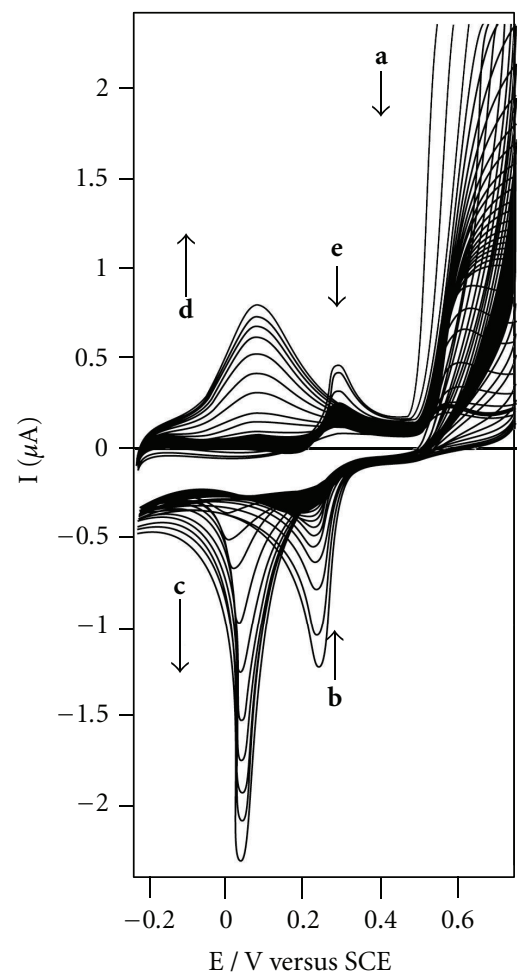

Figure 4: Cyclic voltammograms of $o$ - $\mathrm{AP}$ at $\mathrm{pH} 0.9$ in a solution containing $\mathrm{NaClO}_{4} 0.4 \mathrm{~mol} \mathrm{dm}^{-3}, v=100 \mathrm{mV} \mathrm{s}^{-1}$. Area of the working electrode: $1.9 \times 10^{-3} \mathrm{~cm}^{2}$. Arrows indicate the change in the peak height as the electropolymerization is taking place [12].

presented in Figure 4 with those shown by Barbero et al. in [10] for 3APZ allowed Ortega concluding that the coupling of $3 \mathrm{APZ}$ units to form the polymer is the process that occurs during the propagation of the polymeric chains and then, the peaks $\mathrm{c}$ and $\mathrm{d}$ in Figure 4 should be assigned to the redox reaction of $3 \mathrm{APZ}$ and POAP. Ortega remarks that the charge propagation proceeds slowly during the formation of POAP. The slow diffusion of $o$-AP species through the film to reach the electrode and produce more monocation radical was considered to be the cause by which peaks $\mathrm{a}, \mathrm{b}$, and $\mathrm{e}$ decrease with time, and then, the produced film does not oxidize monomer because it is nonconducting at positive potentials. Also, the fact that the growth of the polymer starts after an induction time long after the peaks corresponding to $o$-AP radical cation and dimer become small was considered by Ortega as a confirmation that the propagation process involves further oxidation and coupling of 3APZ.

The electrochemical synthesis of POAP on the surface of a carbon paste electrode (CPE) in the presence of sodium dodecyl sulfate (SDS) is described by Ojani et al. [13]. These authors show, as Barbero [10] and Ortega [12], that in the absence of SDS, $o$-AP is irreversibly oxidized at positive potential values $(0.7 \mathrm{~V}$ versus $\mathrm{Ag} / \mathrm{AgCl} / \mathrm{KCl} 3 \mathrm{M}$ ) without corresponding cathodic processes in the reverse scan. As in other works, after consecutive cycles, one redox process was observed on the voltammetric response, which was assigned to $3 \mathrm{APZ}$. The peaks corresponding to this process did not 


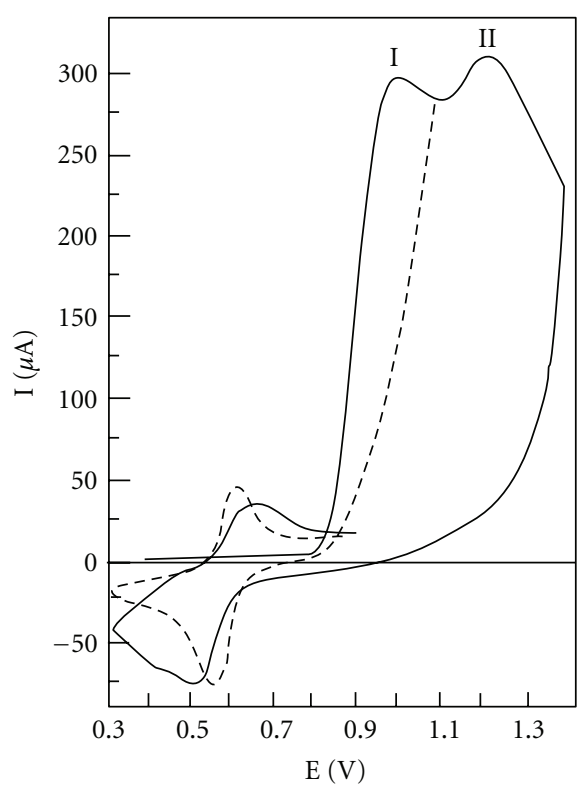

(a)

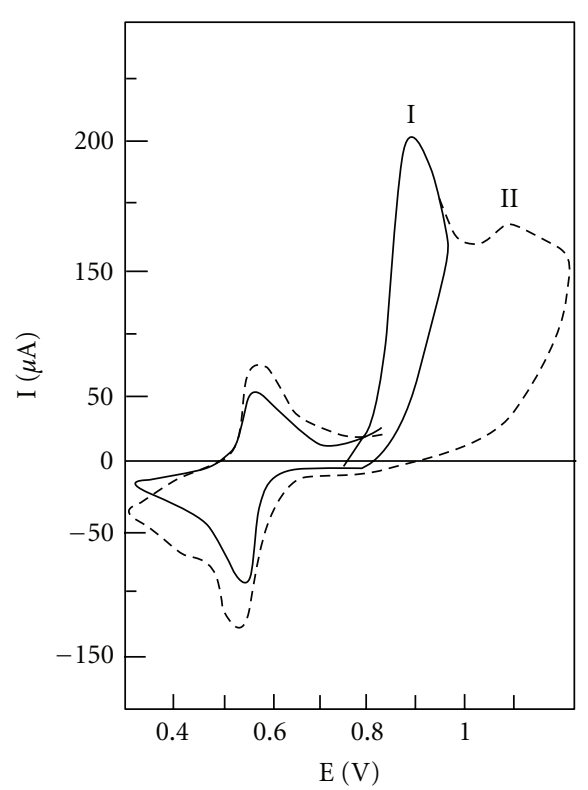

(b)

FIgure 5: Cyclic voltammograms of (a) Pt and (b) GC electrodes in a $0.2 \mathrm{M} \mathrm{NaClO}_{4}+0.1 \mathrm{M} \mathrm{HClO}_{4}+5 \mathrm{mmol} \mathrm{L}^{-1} o$-AP solution. Scan rate $\nu=100 \mathrm{mV} \mathrm{s}^{-1}[14]$.

increase considerably with potential cycling. This effect was attributed by Ojani et al. [13] to soluble products produced on the electrode surface that do not allow the monomer to reach the electrode surface and produce the monocation radical. Therefore, prolonged potential cycling was needed for transformation of the soluble $3 \mathrm{APZ}$ to POAP, which was observed within a potential range $(0.2 \mathrm{~V}<E<0.3 \mathrm{~V})$ less positive than that of $3 \mathrm{APZ}$. When SDS is added to the monomer solution, the monomer oxidation potential was shifted to less positive potentials (almost $0.075 \mathrm{~V}$ ) and the oxidation current increased, as compared with the process in the absence of SDS. The rate of polymerization also increased considerably in the presence of SDS and the peaks attributed to POAP grew simultaneously with that corresponding to $3 \mathrm{APZ}$ growth. It is remarked in [13] that in the presence of SDS the monomer, $o$-AP can easily reach the electrode surface and produce more monocation radical than in absence of SDS. The rate of polymerization depends on the SDS concentration. Up to $0.1 \mathrm{mM}$ of SDS, the rate of polymerization was not much larger than that observed without SDS. However, for SDS concentration values higher than $0.1 \mathrm{mM}$, the rate of polymerization increased rapidly and finally, it saturated at around $4 \mathrm{mM}$. The redox behavior of the polymers synthesized in the absence and in the presence of SDS was also investigated in the supporting electrolyte solution. The comparison of the electrochemical response of the immediately prepared SDS/POAP/CPE electrode with that obtained after immersing the electrode $1 \mathrm{~h} 0.5 \mathrm{M} \mathrm{HClO}_{4}$ allowed to demonstrate that the immediately prepared electrode shows two redox couples at about $0.2 \mathrm{~V}$ and $0.3 \mathrm{~V}$, which were assigned to the phenoxazine units of POAP and $3 \mathrm{APZ}$, res-pectively.

The electrochemical oxidation of $o$-AP from a $0.2 \mathrm{M}$ $\mathrm{NaClO}_{4}+0.1 \mathrm{M} \mathrm{HClO}_{4}$ solution on platinum $(\mathrm{Pt})$ and glassy carbon (GC) electrodes employing cyclic voltammetry, was studied by Gonçalves and coworkers [14]. Cyclic voltammograms of Pt and GC in the electrolyte solution containing $5 \mathrm{mmol}^{-1}$ of $o$-AP are compared in Figure 5. o-AP oxidizes irreversibly on both electrodes, and two anodic peaks (I and II) are observed without corresponding cathodic processes in the reverse scan. Peaks I and II appear for the GC electrode as well-separated waves and at more negative potentials than for $\mathrm{Pt}$, which was attributed to a more favorable process of oxidation on the former electrode material. Peak II was related to followup oxidation reactions after peak I. Besides, the nature of the electrode surface, the characteristics of the films synthesized in [14] depended on variables such as the final potential and the value of the potential scan rate. It was observed that the current of peak I increased linearly with the square root of the scan rate $\left(v^{0.5}\right)$ indicating a process controlled by ion diffusion. The current function $\left(I_{p} \nu^{0.5} c\right)$ versus $v^{0.5}$ plot showed the existence of successive electrochemical-chemical-electrochemical steps $\mathrm{E}(\mathrm{CE})_{n}$ during the $o$-AP oxidation and two electrons were estimated to be involved in the process. Gonçalves and coworkers [14] postulate that besides a film with a ladder structure, the oxidation of $o$-AP can produce intermediate benzoquinone monoamine after successive cycling. It is also remarked in [14] that although APZ is formed in solution after successive cycling, it does not polymerize as proposed by Barbero et al. [10] and Ortega [12]. The electrode material seems to affect the $o$-AP electropolymerization process. In this regard, the redox processes of the POAP films deposited on Pt and GC electrodes in the supporting electrolyte were found to be different. A less electroactive film was obtained on Pt electrodes and compared with GC electrodes.

The effect of the electrode surface on the electropolymerization process of $o$-AP was studied in details in [15]. 

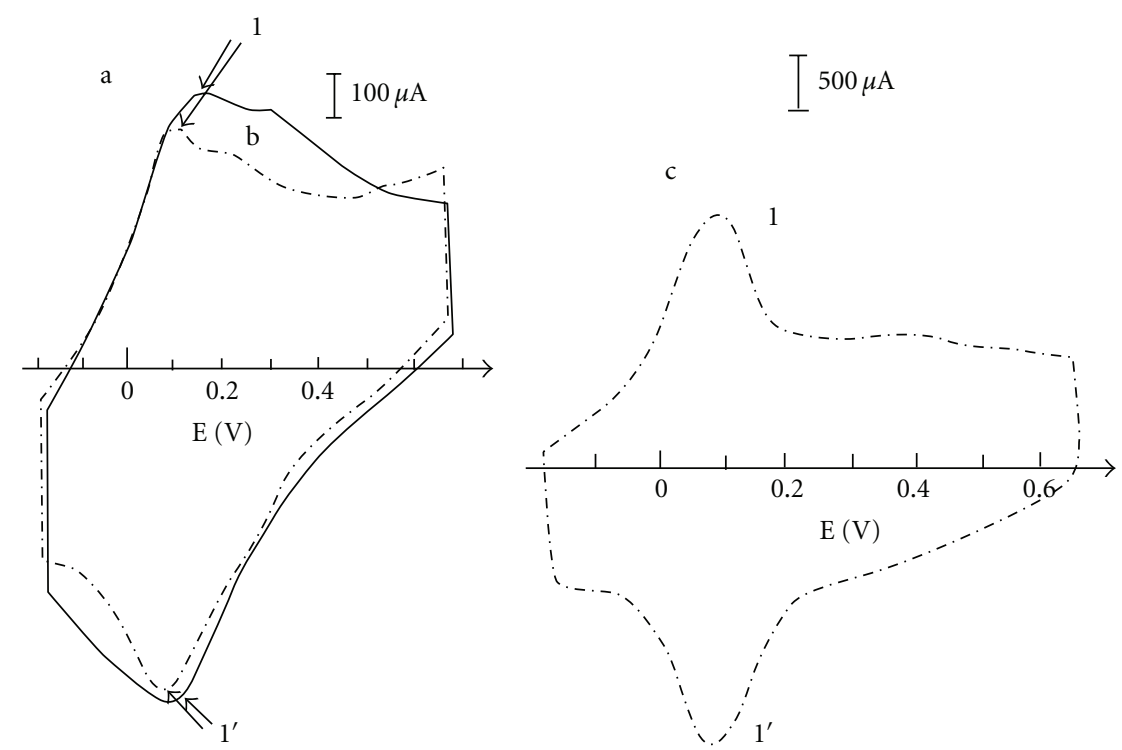

FIGURE 6: Cyclic voltammograms of POAP films $\left(46 \mathrm{mC} \mathrm{cm}{ }^{-2}\right)$ on different carbon electrode substrates in $0.1 \mathrm{M} \mathrm{H}_{2} \mathrm{SO}_{4}$ : (a) original GC electrode, (b) chemically pretreated electrode using $98 \%$ sulphuric acid for $10 \mathrm{~min}$, (c) electrochemically pretreated GC electrode at $1.85 \mathrm{~V}$ for $5 \mathrm{~min}$, then cycled between $-0.2 \mathrm{~V}$ and $0.7 \mathrm{~V}$ for another $15 \mathrm{~min}$ in $0.1 \mathrm{M} \mathrm{H}_{2} \mathrm{SO}_{4}$ [15].

Glassy carbon GC electrodes were chemical and electrochemically pretreated before electropolymerization of $o$-AP. The electrode surfaces were polished carefully to a mirror finish and then cleaned ultrasonically with triply distilled water for $3 \mathrm{~min}$. This kind of electrode was denoted in [15] as an "untreated" electrode. In addition, electrochemical treatment of the electrodes was achieved by placing the polished electrodes in $0.1 \mathrm{M} \mathrm{H}_{2} \mathrm{SO}_{4}$ at $1.85 \mathrm{~V}$ (SCE) for $5 \mathrm{~min}$, and then they were cycled between $-0.2 \mathrm{~V}$ and $0.7 \mathrm{~V}$ for $15 \mathrm{~min}$. Chemical pretreatment of the electrodes was achieved by immersing the electrodes in concentrated nitric acid $(67 \% \mathrm{wt} / \mathrm{wt})$ and sulfuric acid $(98 \% \mathrm{wt} / \mathrm{wt})$ for $10 \mathrm{~min}$, respectively. In most experiments described in [15], cyclic voltammetry was employed to synthesize POAP films, using a potential sweep rate of $100 \mathrm{mV} \mathrm{s}^{-1}$. Electropolymerizations were carried out on these different surfaces by cycling the electrode potential between $-0.2 \mathrm{~V}$ and $0.7 \mathrm{~V}$ and the net charge passed in each kind of electrode was assessed. As was proposed by Barbero et al. [10], a composite of two different (ladder-like and linear-chain) structures was considered to be formed on the electrode surfaces under the polymerization conditions employed in [15]. Figures 6(a), 6(b), and 6(c) compare three stable cyclic voltammograms of POAP films on untreated, chemically and electrochemically pretreated GC electrode surfaces, respectively. The net charge during the electropolymerization process of each polymer film was $46 \mathrm{mC} \mathrm{cm}^{-2}$. The oxidation current peak 1 and the reduction current peak $1^{\prime}$ were attributed to the redox reaction of the polymer film. The highest peak current was obtained for the electrochemically treated GC electrode (Figure 6(c)). The peak current value for the film formed on the electrochemically pretreated electrode is about four times that of the untreated electrode. The reversibility of the redox reaction is also improved and the ratio $I_{\mathrm{pa}} / I_{\mathrm{pc}}$ is nearer to unity in the electrochemically pretreated electrode. These differences were attributed, in part at least, to the formation of thicker POAP films on the pretreated electrode due to chemical oxidation of $o$-AP by some oxide species present on the surface electrode after pretreatment. $\mathrm{C}_{n}{ }^{+} \mathrm{HSO}_{4}{ }^{-}$species on the GC surface was considered as possible oxidant and/or newly formed oxide species after electrochemical pretreatment. On the other hand, the authors of [15] also verified that the electron-transfer rate from the polymer film to the electrode substrate on electrochemically pretreated GC electrodes was at least twice that of the electron-transfer rate for the untreated electrode. It was proposed that some functional group, such as quinone-like species, may serve as an "electron mediator" at the polymer-solution interface. The effect of the pretreatment potential on the electropolymerization rate of $o$-AP was also analyzed in [15]. The electropolymerization rate on the pretreated electrode was not very different from that on the untreated electrodes when the pretreatment potential was $1.55 \mathrm{~V}$. However, it was observed that for pretreatment potentials of $1.85 \mathrm{~V}$ and $2.05 \mathrm{~V}$, respectively, the electropolymerization rate of the monomer increased with the increase of the pretreatment potential. The faster polymerization rate at higher pretreatment potentials was attributed to the higher amounts of surface oxide species formed on the electrode surface. This study carried out in [15] on different GC surfaces seems to indicate that oxide species formed on the electrode surface can play the role of oxidants within the electropolymerization process of $o$-AP and these species oxidize $o-\mathrm{AP}^{\bullet+}$ to the oligomer of $o$-AP. With regard to chemical pretreatment of the electrodes, the authors of [15] remarked that external reflection FT-IR spectra and attenuated total reflection (ATR) FT-IR spectra showed evidence that nitro groups are formed on carbon after chemical treatment with nitric acid. Then, the effect of nitro and 
sulfate groups present at the surfaces of chemically pretreated GC electrodes on the electropolymerization of $o$-AP was also analyzed in [15]. It was observed that the electropolymerization rate for the electrodes which were treated with nitric acid was quite slow, even slower than the rate for untreated electrodes. In this regard, a polymer film whose charge was $46 \mathrm{mC} \mathrm{cm}^{-2}$ was obtained in about $40 \mathrm{~s}$ on electrochemically pretreated GC electrodes. On both, untreated and sulfuric acid-treated electrodes, the formation of a similar thickness of film took about $5 \mathrm{~min}$. However, only a $23 \mathrm{mC} \mathrm{cm}^{-2}$ thickness film was obtained on nitric-acid-treated electrodes after $30 \mathrm{~min}$ of polymerization. All these findings allowed the authors of [15] concluding that the different surface species present on different chemically pretreated GC electrodes may have different effects on the electropolymerization process of $o$-AP and then, different electropolymerization mechanisms may also be involved in the formation of POAP.

Kunimura and coworkers [16] prepared POAP on basalplane pyrolytic graphite (BPG) and In-Sn oxide conducting glass (ITO) by electrooxidative polymerization of $o$-AP. The electrode potential was cycled between -0.4 and $1.0 \mathrm{~V}$ (versus a sodium chloride saturated calomel electrode) at $50 \mathrm{mV}$ $\mathrm{s}^{-1}$ in a $0.5 \mathrm{M} \mathrm{Na}_{2} \mathrm{SO}_{4}$ solution ( $\mathrm{pH}$ 1) containing $50 \mathrm{mM}$ $o$-AP. As reported in other works $[10,12,14,24]$, it was observed that $o$-AP electrooxidizes irreversibly at high positive potentials and after the first reverse scans, anodic and cathodic currents are developed within the potential region comprised between -0.2 and $0.2 \mathrm{~V}$. The current response within this last potential region increased continuously with successive potential scans, indicating the buildup of the electroactive polymeric product POAP. As the potential scan was continued, both electrode (BPG and ITO) surfaces were covered with a brownish orange thin film. A reversible redox response of the POAP film was observed in the only presence of the supporting electrolyte solution $\left(0.2 \mathrm{M} \mathrm{NaClO}_{4}, \mathrm{pH}\right.$ 1). The amount of charge involved in both oxidation and reduction processes was found to be almost equal. POAP films obtained in [16] were characterized by IR spectroscopy and the spectra of POAP were compared with those of $o$-AP and phenoxazine. From this comparison, two structures of POAP were proposed: a partially ring-opened structure (Figure 7) and another partially hydrolyzed (Figure 8, see Section 2).

Zhang et al. [17] studied the electrochemical synthesis of POAP on GC in a $1 \mathrm{M} \mathrm{SO}_{4} \mathrm{H}_{2}+0.5 \mathrm{M} \mathrm{Na}_{2} \mathrm{SO}_{4}$ solution by cyclic voltammetry and Raman spectroscopy. The evolution of the cyclic voltammograms during polymerization of $o$-AP within the potential range comprised between $-0.2 \mathrm{~V}$ and $1.0 \mathrm{~V}$ (SCE) was analyzed in [17]. Three redox pairs were observed. The most negative redox pair was observed at around $0-0.15 \mathrm{~V}$ and it was the only noticeable feature in the cyclic voltammogram of POAP in an acid medium without the monomer. While this redox pair was attributed to the redox reactions of $o$-AP polymers and/or oligomers, the other two more positive peak systems were associated to the oxidation of $o$-AP to the radical cation $\left(\mathrm{OAP}^{\bullet+}\right)$ and its further oxidation to the dication, respectively. While the peaks of the redox pair at $0-0.15 \mathrm{~V}$ increased steadily with increasing the number of scans, showing the gradual but<smiles>Cc1ccc(Oc2cc3nc(C)c(C)oc-3cc2=N)cc1C</smiles>

Figure 7: Partially ring-opened structure of POAP [16].<smiles>CN=C1C=CC(N=C2C=CC(N=C3C=CC(C)=CC3=O)=CC2=O)=CC1=O</smiles>

FIGURE 8: Partially hydrolyzed structure of POAP [16].

continual formation of electroactive POAP, the peaks of the other two redox pairs decreased. The flattening of the two more positive peak systems was attributed to a limitation on $o$-AP diffusion during the polymerization process. The scarce formation of the radical cation (polaron) and dication (bipolaron) during the polymerization was associated to restricted charge transport processes and electron delocalization effects along a partly cross-linked polymer chain. It was proposed in [17] that POAP is formed predominantly by the attack of oxidized monomers on the polymer growing chain. In situ Raman spectroscopy measurements performed in [17] suggest that the POAP matrix contains alternating oxidized (quinonoid) and reduced (N-phenyl- $p$-phenylenediamine) repeating units (Figure 9, see Section 2).

Zhang and coworkers in another work [18] synthesized POAP on GC and Pt electrodes from a solution containing $0.05 \mathrm{M} o$-AP in a mixture of $1 \mathrm{M} \mathrm{H}_{2} \mathrm{SO}_{4}$ and $0.5 \mathrm{M} \mathrm{Na}_{2} \mathrm{SO}_{4}$ to study the oxidation process of POAP deposits of different thicknesses. Potential was scanned from $-0.2 \mathrm{~V}$ to $0.8 \mathrm{~V}$ (SCE) at $100 \mathrm{mV} \mathrm{s}^{-1}$ for different numbers of cycles $(N)$. The extent of the oxidation of the polymer films was investigated at the open-circuit potential of POAP. The synthesis conditions employed in [18] allowed the authors to propose the same POAP structure formulated in [17] (Figure 9). The relative proportions of alternating oxidized (quinonediimine) and reduced (phenylenediamine) repeating units were found to be dependent on the oxidation state of the polymer. Although the polymer synthesized in [17] corresponds to the reduced state $(E=-0.2 \mathrm{~V})$ of POAP, it was observed in [18] that it is readily oxidized by dissolved oxygen in the electrolyte and the extension of the oxidation depends on the film thickness. Thick films were more difficult to oxidize and often result in a mixture of reduced and oxidized forms. With the diffusion of oxygen impeded by the increase of the film thickness, oxidation of thick POAP films was confined mostly to the polymer exterior. The structure of POAP proposed in [18] allowed also the authors explaining the interaction of the polymer with metal cations. In this regard, it was observed that when POAP films deposited from 150 voltammetric cycles are equilibrated in a $0.1 \mathrm{M} \mathrm{AgNO}_{3}$ solution for $30 \mathrm{~min}$, the films capture silver cations. The cation capturing process was attributed to simultaneous presence of hydroxyl and amino groups of the polymeric backbone of POAP, in which the lone pair electrons are available to 


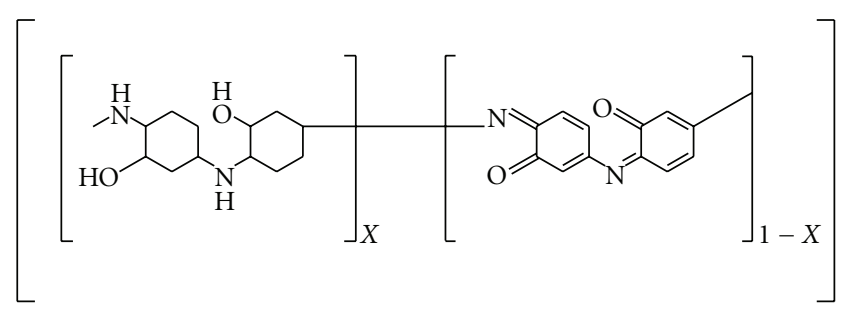

FIgURE 9: Structure of POAP as an alternating series of oxidized (quinonoid) and reduced (N-phenyl-p-phenylenediamine) repeating units [17].

coordinate with metal cations (Figure 9). The interaction of POAP with silver ions ranged from redox reactions, in which cations were reduced to the metallic form, to a partial charge transfer between the metal and the polymer resulting in the formation of a metal-polymer complex. These two types of interaction predominate in thick films $(N=120)$ and thin films $(N=1)$, respectively. Films of moderate thickness $(N=20)$ exhibited intermediate behavior. The $\mathrm{Ag}^{+}$-POAP complex synthesized in [18] was also compared with that of Ag-PANI [25]. It is indicated that the $\mathrm{Ag}^{+}$-POAP complex presents an improved stability over that of $\mathrm{Ag}^{+}$-PANI due to the cooperative action of the oxygen atom in the POAP chemical structure. Besides, the redox reaction of silver is within the range of the POAP redox reaction, and then, changes of POAP conductivity were not significant during the redox reactions of the POAP- $\mathrm{Ag}^{+}$complex. This was very different from the situation of the $\mathrm{Ag}^{+}$-PANI complex, where the redox switching of PANI between the insulating state of leucoemeraldine and the conducting state of emeraldine has substantial influence on the voltammetric response of silver redox behavior. Also, it has also been demonstrated in [18] that POAP is more resistant than PANI to electrochemical degradation and can capture four times much silver than PANI. POAP-Ag(I) complex also exhibits electrocatalytic activity in dissolved oxygen reduction. However, it was observed that silver can be released from the complex upon acidification of the nitrogen and oxygen atoms or upon application of a sufficient positive potential.

The 1,4-substituted molecular structure of POAP proposed by Zhang et al. [17, 18] (Figure 9) also seems to be consistent with the electrochemical response of POAP films to ferric cation in solution $[26,27]$. It was reported in [26] that POAP films obtained on ITO electrodes by electropolymerization of $o$-aminophenol $(0.1 \mathrm{M})$ in a $0.1 \mathrm{M} \mathrm{H}_{2} \mathrm{SO}_{4}$ aqueous solution, after being soaked in a ferric cation solution, can act as potentiometric Fe(III) ion sensors. In this regard, POAP films immediately synthesized in [26] showed IR spectra that correspond to a 1,4-substituted structure. When these POAP films are soaked for $24 \mathrm{~h}$ in a $0.1 \mathrm{M} \mathrm{H}_{2} \mathrm{SO}_{4}$ aqueous solution containing $50 \mathrm{mM} \mathrm{Fe}_{2}(\mathrm{SO} 4)_{3}$, their XPS spectra showed iron ion capture. The ferric cation capturing process was attributed to simultaneous presence of hydroxyl and anino groups of the polymeric backbone of POAP. After this cation capture process, the electrode potential of the POAP film was measured in various aqueous solutions containing $\mathrm{Zn}(\mathrm{II}), \mathrm{Ni}(\mathrm{II}), \mathrm{Cu}(\mathrm{II}), \mathrm{Fe}(\mathrm{II})$, and $\mathrm{Fe}(\mathrm{III})$ ions at different concentrations. The relationship between the electrode potential, $E$, and the logarithm of the concentration,
$C$, in the different cations in solutions was recorded. The electrode showed no potential response to ion concentration for $\mathrm{Zn}(\mathrm{II}), \mathrm{Ni}(\mathrm{II}), \mathrm{Cu}(\mathrm{II})$, and $\mathrm{Fe}(\mathrm{II})$ ions. However, it showed a Nernstian potential response to $\mathrm{Fe}(\mathrm{III})$ ions with a slope $-57 \mathrm{mV} / \log [\mathrm{Fe}(\mathrm{III})]$. The response time was less than $10 \mathrm{~s}$, and the response was observed until $[\mathrm{Fe}(\mathrm{III})]=10^{-4} \mathrm{M}$. The response to $\mathrm{Fe}$ (III) ions in solution was considered indicative of the presence of Fe(II) in the POAP film. The presence if $\mathrm{Fe}$ (II) into the film was explained considering that the captured $\mathrm{Fe}$ (III) are, at least in part, reduced to $\mathrm{Fe}$ (II) by the film. The potentiometric response was attributed to the electron transfer between $\mathrm{Fe}(\mathrm{II})$ ions into the film and $\mathrm{Fe}$ (III) ions in solution.

Despite the extensive electrochemical studies of POAP potentiodynamically synthesized, Shah and Holze [19] investigated the potentiostatic electrochemical polymerization of $o$-AP at different electrode potentials $(E=0.7 \mathrm{~V}, 0.8 \mathrm{~V}$, and $0.9 \mathrm{~V}$ versus SCE, resp.) with the aim to compare the redox behaviors of the synthesized polymer by cyclic voltammetry and potentiostatically. POAP films potentiostically synthesized at $0.7 \mathrm{~V}$ show two redox processes. The first redox process is centered at $E=0.16 / 0.15 \mathrm{~V}$ (SCE) while the second one is observed at $E=0.35 / 0.29 \mathrm{~V}$. The contribution of the second redox process decreases as the potential applied during the electrosynthesis is increased. The voltammogram of POAP obtained at $E=0.90 \mathrm{~V}$ presents a somewhat intermediate behavior between that of films obtained potentiostatically at $E=0.7 \mathrm{~V}$ and film potentiodynamically synthesized. In order to interpret the second redox process at POAP films potentiostatically synthesized, dependences of the cyclic voltammogram upon potential scan rate and solution $\mathrm{pH}$ were analyzed in [19]. With regard to potential scan rate, both the anodic and cathodic peak currents of the first redox process were found to scale linearly with the potential sweep rate in the range $10 \mathrm{mV} \mathrm{s}^{-1}<v<100 \mathrm{mV} \mathrm{s}^{-1}$, indicating that the electrochemical process of POAP is a surface process and kinetically controlled. The second redox process was not well defined to apply the same analysis. With regard to the $\mathrm{pH}$ effect, by increasing the solution $\mathrm{pH}$ from 0 to 1.0 , the first redox-couple remained almost unaffected while the peak currents of the second redox pair were decreased. While the first redox pair showed a little change up to $\mathrm{pH}$ 2.0 , the second redox pair was affected to a great extent as the anodic peak was replaced by a shoulder and the cathodic peak by a current plateau. By further increase of the solution $\mathrm{pH}$ to 3.0, the first redox pair was further shifted towards negative potentials but the second pair was almost completely replaced by anodic and cathodic current plateaux. 
Beyond $\mathrm{pH}$ 3, the first redox couple was also affected very much. Raman and UV-Vis spectra were also recorded in [19] to differentiate potentiostatically and potentiodynamically synthesized POAP films. Although Raman and UV-Vis spectra of POAP films potentiostatically and potentiodynamically synthesized were found to be similar, some differences were observed in the dependence of the bands intensities on the applied potential (see Section 2). These differences were assigned to the ladder-type polymer structure. Raman and UV-Vis spectroscopic measurements reveal that the redox transition of potentiostatically synthesized POAP from the completely reduced state to the completely oxidized one proceeds through two consecutive reactions in which a charged intermediate species takes part. Then, in the light of electrochemical and spectroscopic analysis carried out in [19], a mechanism for the redox transformation of potentiostatically prepared POAP films was proposed, in which phenoxazine units take part.

The development of an NO sensor based on hybrid films of POAP and the metal complex Ni-sulfonated phtalocyanine is reported in [20]. POAP films were produced by cycling the base electrode (Pt or GC) between -0.25 and $0.7 \mathrm{~V}$ (SCE) at $50 \mathrm{mV} \mathrm{s}^{-1}$ in a $0.05 \mathrm{M} \mathrm{o}$-aminophenol solution in $0.5 \mathrm{M}$ $\mathrm{HClO}_{4}$. To incorporate the metal complex, sulfonated nickel phtalocyanine was added to the solution at a $10 \mathrm{mM}$ concentration. The polymerization in the presence of the metal complex is faster than with $o$-aminophenol alone. This fact was attributed to a template effect of the sulfonic groups. After several cycles $(>50)$, a film of POAP-sulfonated nickel phtalocyanine (POAP/NiSuPh) was deposited on the electrode. The incorporation of the metal complex into the polymer film was tested by ex-situ reflection-absorption FTIR spectroscopy. The hybrid film, produced with the metal complex, revealed new bands at 1063 and $1030 \mathrm{~cm}^{-1}$, with respect to those corresponding to POAP. These bands were assigned to stretching of the sulfonate group. The bands are retained after cycling in a solution without the complex, even at $\mathrm{pH}$ 7. The modified electrodes were tested for NO electrochemical oxidation. A peak at $0.95 \mathrm{~V}$ was observed on the cyclic voltammogram that corresponds to $\mathrm{NO}$ oxidation, and it increases with $\mathrm{NO}$ concentration. It was observed that both POAP and POAP/NiSuPh films present higher electrocatalytic activity for $\mathrm{NO}$ oxidation than the base substrate (GC). The oxidation current, in the electrode modified with the complex, is higher and the oxidation overpotential is lower, indicating that the $\mathrm{Ni}$ complex incorporated into the hybrid film could electrocatalyze NO oxidation. The peak current was measured to determine the concentration of $\mathrm{NO}$ in the solution. The peak current is linear with a concentration up to $200 \mu \mathrm{M}$.

The electro-oxidation of methanol in aqueous acid solutions using GC electrodes modified by a thin film of POAP containing dispersed platinum and platinum alloy microparticles was studied in [21]. Pt, Pt-Ru, and Pt-Sn particles were incorporated into the polymer film by electrochemical deposition and the following electrodes were synthesized: GC/POAP/Pt, GC/POAP/Pt-Ru, and GC/POAP/Pt-Sn. A mean value of $0.52 \pm 0.02 \mu \mathrm{m}$ was employed for the polymer film thicknesses in the different electrodes. Electropolymerization of $o$-AP was performed either by potentiodynamic or potentiostatic methods. In potentiodynamic procedure, the electrode potential was swept between $-0.10 \mathrm{~V}$ and $1.00 \mathrm{~V}$ (SCE) at a scan rate of $20 \mathrm{mV} \mathrm{s}^{-1}$. In potentiostatic method, the electrode potential was fixed at $1.0 \mathrm{~V}$ for a given time. In both cases, an aqueous solution containing $0.10 \mathrm{M} \mathrm{HClO}_{4}$ and $0.10 \mathrm{M} o$-AP was used as electrolyte. In all experiments, the working electrode was submerged in open-circuit position into methanol solutions. Then, voltammograms were recorded by sweeping the potential from $0.0 \mathrm{~V}$ to $1.00 \mathrm{~V}$ (versus SCE). Although the Pt loading for both electrodes (GC/Pt and GC/POAP/Pt) was similar, it was observed that the peak current for the oxidation of methanol $(0.63 \mathrm{~V}$ versus SCE) increased from $0.48 \mathrm{~mA}$ (GC/Pt electrode) to $1.73 \mathrm{~mA}$ (GC/POAP/Pt; area of the GC electrodes: $0.07 \mathrm{~cm}^{2}$ ). This result was explained in terms of an enhancement of $\mathrm{Pt}$ microparticle efficiency toward the catalytic oxidation of methanol in the presence of POAP. Similar results were also obtained when Pt was replaced by Pt-Sn or Pt-Ru alloys. Chronoamperometric experiments indicated the existence of an increase in the real surface area of a GC/POAP/Pt electrode with respect to that of a GC/Pt electrode (the area of the $\mathrm{GC} / \mathrm{POAP} / \mathrm{Pt}$ electrode was 2.85 times larger than that of the $\mathrm{GC} / \mathrm{Pt}$ electrode). Then, an augmentation of the real surface was invoked in [21] to explain the increase in reactivity of the electrocatalyst deposited in the presence of POAP. Thus, the role of the polymeric matrix is not directly connected with an increase of the intrinsic specific activity of the electrocatalyst, but the polymer film only acts as a good and proper bed for deposition of electrocatalyst particles and increases the catalytic activity of active sites.

2.2. Electropolymerization of o-Aminophenol in Alkaline and Neutral Media. The electropolymerization of $o$-AP from a $0.3 \mathrm{M} \mathrm{NaOH}$ hydroalcoholic solution $\left(70 \mathrm{vol} \% \mathrm{H}_{2} \mathrm{O}, 30 \mathrm{vol} \%\right.$ $\mathrm{CH}_{3} \mathrm{OH}$ ) containing $0.1 \mathrm{M}$ o-AP to produce a protective polymer layer on different substrates (vitreous carbon, platinum, and copper) is described in [22].

Figures $10(\mathrm{a}), 10(\mathrm{~b})$, and 10(c) show cyclic voltammetry curves obtained, respectively, for vitreous carbon (VC), platinum $(\mathrm{Pt})$, and copper in the $0.3 \mathrm{M} \mathrm{NaOH}$ hydroalcoholic solution free of monomer (dotted line). The oxidation leads to an increase of the current near $0.3 \mathrm{~V}$ (SCE). The addition of $0.1 \mathrm{M}$ of $o$-AP to the hydroalcoholic solution (continuous line) induced a current peak with a maximum at, respectively, $0.09 \mathrm{~V}(\mathrm{VC}), 0.16 \mathrm{~V}(\mathrm{Pt})$, and $0.2 \mathrm{~V}$ (copper). This peak was attributed to the oxidation of $o$-AP that started at $-0.2 \mathrm{~V}$ at $\mathrm{pH} 12$. In the case of $\mathrm{VC}$ and $\mathrm{Pt}$ substrates (Figures 10(a) and 10(b), resp.), the peak of current was followed by a wide passivity domain, characterized by a very weak current, showing the formation of a very protective film. After the forward part, the reverse curve with negligible current confirmed the insulating character of the film and showed the irreversibility of the system. Figure 10(c) illustrates the behavior of the copper substrate. The forward part of the curve for copper presents a second peak at a high potential $(0.765 \mathrm{~V}$ versus SCE), attributed to the substrate oxidation through the holes of the growing film. To confirm 


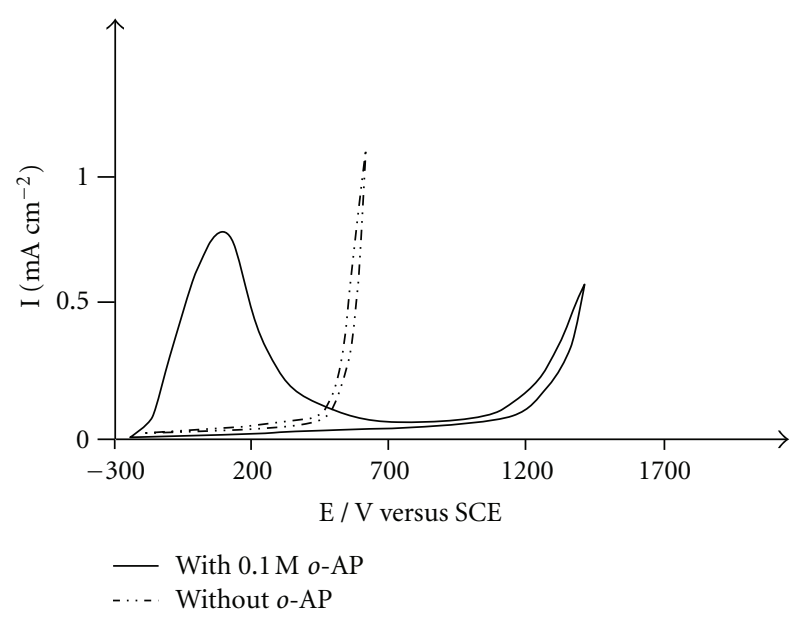

(a)

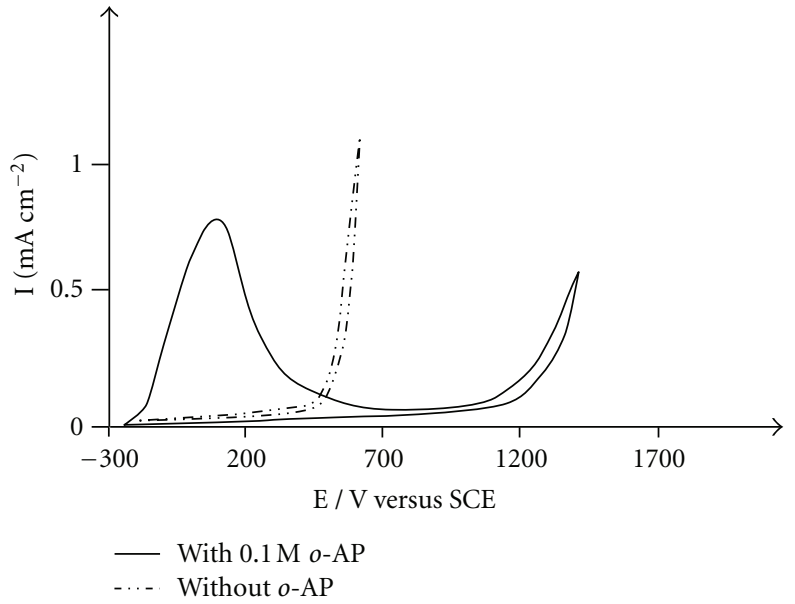

(b)

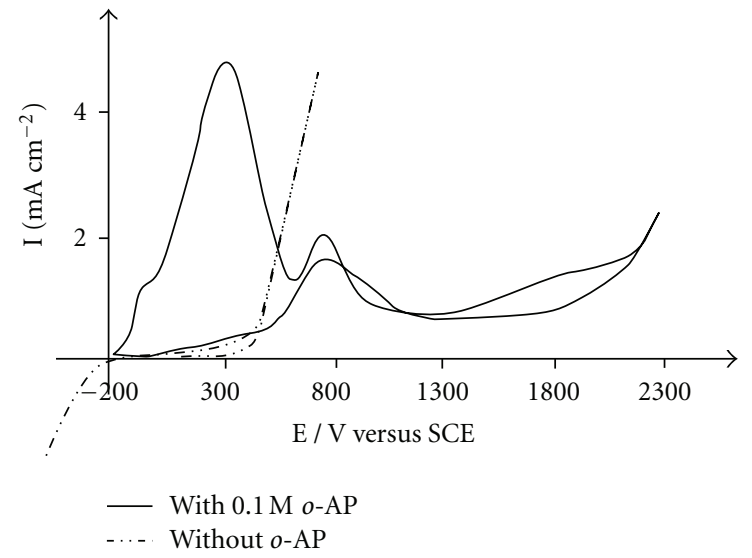

(c)

Figure 10: Voltammograms obtained in $0.3 \mathrm{M} \mathrm{NaOH}$ hydroalcoholic solutions ( $\mathrm{pH}$ 12.7) for (a) Vitreous carbon, (b) platinum, and (c) copper (-) with $0.1 \mathrm{M} o$-AP and (- - -) without $o$-AP. Scan rate $30 \mathrm{mV} \mathrm{s}^{-1}$ [22].

this assertion, the polarization curve was stopped before the apparition of the second peak, the reverse curve shows the presence of the protective film. However, as the voltammetric scan was extended toward the second peak domain, the voltammetric response did not show the formation of a protective layer. The films obtained within the first potential domain, between $-0.2 \mathrm{~V}$ and $0.65 \mathrm{~V}$, were very adherent. However, films obtained on copper in the second potential domain were not adherent, which was attributed to the oxidation products of the substrate mixed with the organic film. Chronoamperometric studies were also carried out in [22]. For constant potential values within the first peak domain, below $0.65 \mathrm{~V}$, a progressive decrease of current densities for the three substrates was observed. Stationary currents were achieved after $20 \mathrm{~min}$. This behavior confirmed the insulating character of the film. For copper polarized at $0.51 \mathrm{~V}$ (near the maximum potential peak or slightly higher), layers very uniform and adherent were obtained. In the case of copper substrate, the electrochemical treatment at high potentials corresponding to the second peak of the polarization curve led to a very different behavior. It was observed that, during a treatment at $0.76 \mathrm{~V}$, current densities have high values, which were attributed to the corrosion phenomenon. The analysis of the electrolyte by atomic absorption revealed the presence of copper ions in the solution which confirmed the dissolution of the substrate. The physical characteristics of the film deposited on copper were dependent on the concentration ratio $R=[\mathrm{NaOH} / o-\mathrm{AP}]$. Results reported in [22] refer to hydroalcoholic solutions containing $0.3 \mathrm{M} \mathrm{NaOH}$ and $0.1 \mathrm{M}$ $o$-AP $(R=3)$ and the films obtained were homogeneous and very adherent. However, the authors remark that with $R=1$, the films were not adherent and presented cracks and large pores. POAP layers deposited on the copper substrate (four cycles between -0.23 and $1.2 \mathrm{~V}$ ) were also analyzed in [22] by employing IR and XPS spectrometry techniques (see Section 2).

Thin nonconducting POAP films synthesized in neutral media are also employed as protective layers for biosensor applications. In general, POAP films are simultaneously synthesized with different electroactive materials such as, hemoglobin, quinhydrone, carbon nanotubes, prussian blue, and other polymers such as poly(o-phenylenediamine) and polypyrrole, which act as electron mediators [18, 23, 28-37], to build different biosensors. POAP is also widely used as 


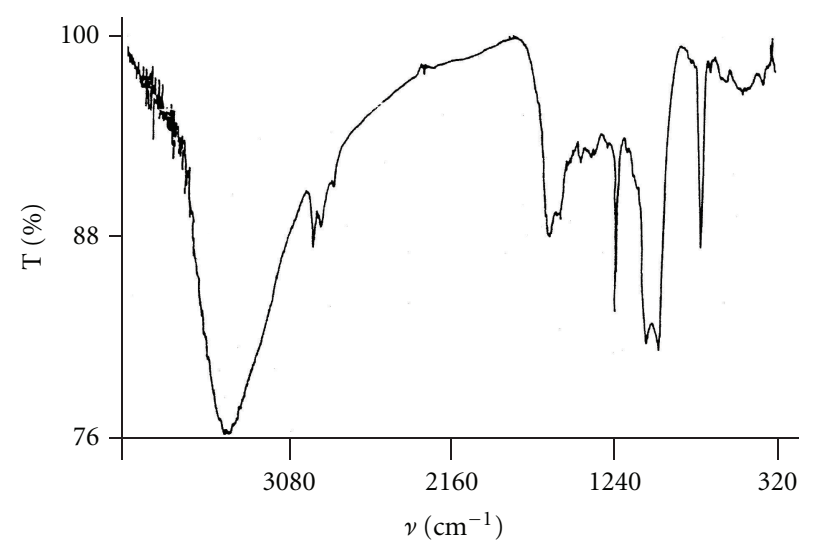

(a)

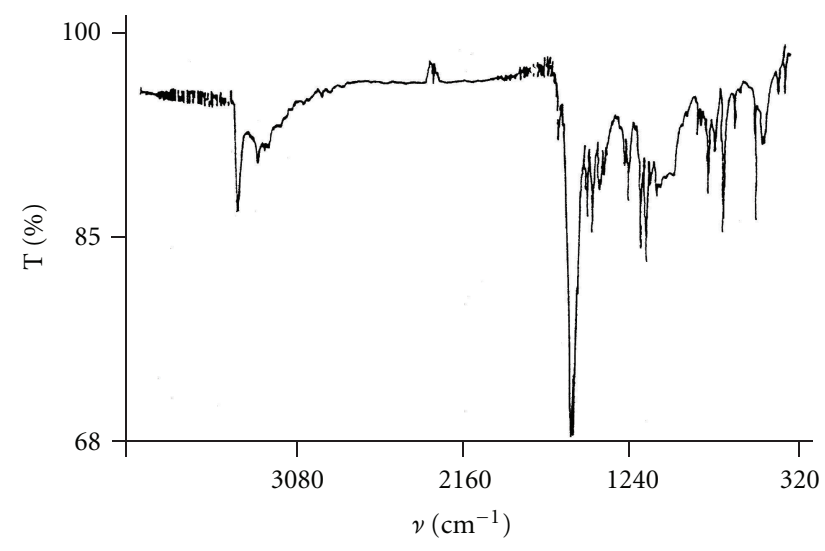

(b)

FIGURE 11: IR spectra of (a) electrochemically prepared POAP, (b) 3-aminophenoxazone [10].

an enzyme matrix in the construction of hydrogen peroxide biosensors because of its catalytic properties toward hydrogen peroxide reduction and limited permeability to small organic molecules [29-32, 36, 37]. An interesting review about synthesis and applications of nonconducting POAP films in bioelectrochemistry and electrocatalysis is found in [38].

\section{The Structure of Electrochemically Synthesized Poly(o-Aminophenol) (POAP) Films Studied by Spectroscopic Methods: The Oxidation Products of Ortho-Aminophenol (o-AP) and the Redox Process of POAP}

Besides electrochemical methods, knowledge about the structure of POAP in both acidic and basic media was obtained by application of different spectroscopic techniques. The study of the redox conversion of POAP employing spectroscopic analysis has also greatly contributed to elucidate the structure of the polymer.

\subsection{Spectroscopic Characterization of POAP Films Synthesized} in Acid Medium. Characterization of electrochemically synthesized POAP films in acid medium employing IR and UVVisible spectroscopy was carried out by Barbero et al. [10]. These authors compare the IR spectrum of an electrochemically prepared POAP film with that of 3-aminophenoxazone (Figure 11). The broad band centered at $3400 \mathrm{~cm}^{-1}$ was assigned to the stretching of the $\mathrm{N}-\mathrm{H}$ bonds, while the bands at 1350 and $1240 \mathrm{~cm}^{-1}$ were ascribed to the $-\mathrm{C}-\mathrm{N}-\mathrm{C}-$ stretching of the secondary amines (Figure 11(a)). Although the stretching of the $\mathrm{C}=\mathrm{N}$ bond was observed at 1633,1462 , and $1384 \mathrm{~cm}^{-1}$, some of these bands were also assigned to the $\mathrm{C}-\mathrm{C}$ bond. The characteristic $=\mathrm{C}-\mathrm{C}=\mathrm{O}$ stretching in $3 \mathrm{APZ}$ at $1573 \mathrm{~cm}^{-1}$ (Figure 11 (b)) only appears as a weak absorption in the POAP. This band in POAP was assigned to a terminal group. The $\mathrm{C}-\mathrm{O}\left(1201 \mathrm{~cm}^{-1}\right)$ and the $-\mathrm{C}-$ $\mathrm{O}-\mathrm{C}$ symmetric stretching are very well defined in POAP. The peak at $800 \mathrm{~cm}^{-1}$ was assigned to the $-\mathrm{C}-\mathrm{H}$ bending vibration of aromatic ortho-substituted aromatic rings. The peak at $1092 \mathrm{~cm}^{-1}$ was ascribed to the $\mathrm{ClO}_{4}{ }^{-}$band. However, this last peak was associated to an impurity because it disappeared after exhaustive washing of the film. Barbero et al. [10] remarked the absence of the characteristic strong absorption of carbonyl group $\left(1680 \mathrm{~cm}^{-1}\right)$ and phenol group $\left(2600 \mathrm{~cm}^{-1}\right)$ in the POAP spectrum. The absence of the carbonyl band was considered to be an indication of an insignificant quantity of $o$-quinone in the film. In the same way, the absence of an important hydroxyl group absorption band was attributed to a low proportion of a linear chain polymer structure (Section 3). POAP structure was also studied by in-situ UV-visible spectroscopy in [10]. A broad maximum around $480 \mathrm{~nm}$ was observed in the oxidized state of POAP. As the UV-Vis spectrum has the same characteristics of that reported for $3 \mathrm{APZ}$, it was considered as a confirmation that the film is constituted of phenoxazine-like units. Then, on the basis of the IR and UV-Visible spectroscopic analysis, the structures shown in Figure 3 were proposed for POAP in the reduced state and in the oxidized one, respectively.

The IR absorption spectrum of the oxidized form of POAP was compared with those of $o-\mathrm{AP}$ and phenoxazine in [16] (Figures 12(a), 12(b), and 12(c), resp.). Despite common absorption peaks for the three compounds were observed, other peaks were not common. The absorption peaks due to the $\mathrm{N}-\mathrm{H}$ stretching vibrations of the imino group of the POAP film and phenoxazine were observed at $3420 \mathrm{~cm}^{-1}$, while two absorption peaks corresponding to the $\mathrm{N}-\mathrm{H}$ stretching vibrations of the amino groups of $o$-AP were, as expected, observed at 3340 and $3420 \mathrm{~cm}^{-1}$. The presence of a relatively strong absorption peak around $3420 \mathrm{~cm}^{-1}$ was considered as an indication that POAP does not possess a completely ring-closed structure (Figure 3 ), as proposed in [10]. In this regard, a partially ring-opened structure as that shown in Figure 7 and/or a relatively low degree of polymerization of $o$-AP was proposed in [16]. The absorption peaks ascribable to the stretching vibrations of $\mathrm{C}-\mathrm{N}$ bonds were observed for POAP at 1250 and $1310 \mathrm{~cm}^{-1}$. Similar peaks were observed for $o$-AP and phenoxazine. The peak 


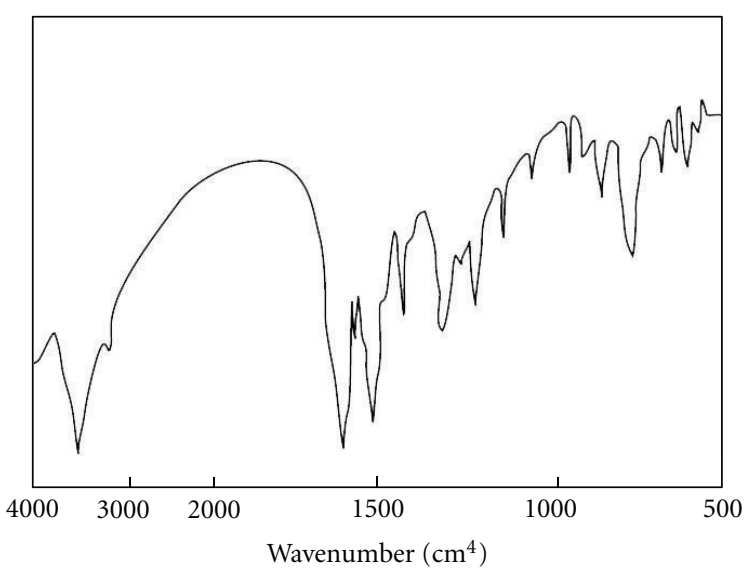

(a)

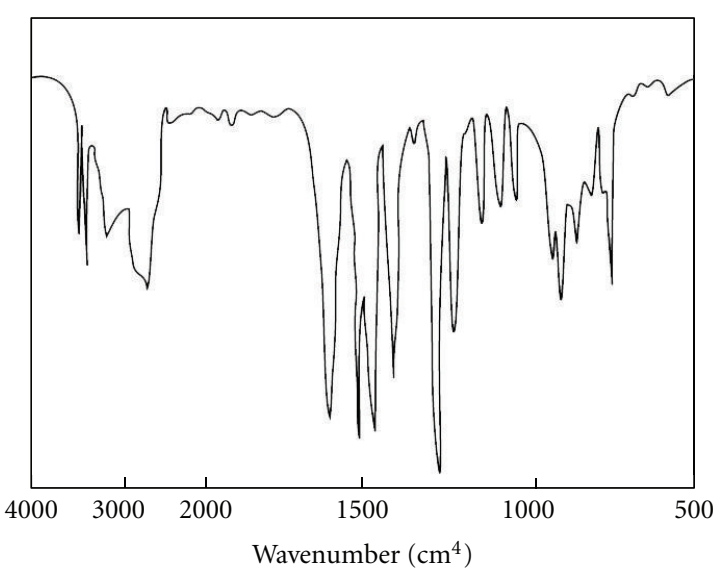

(b)

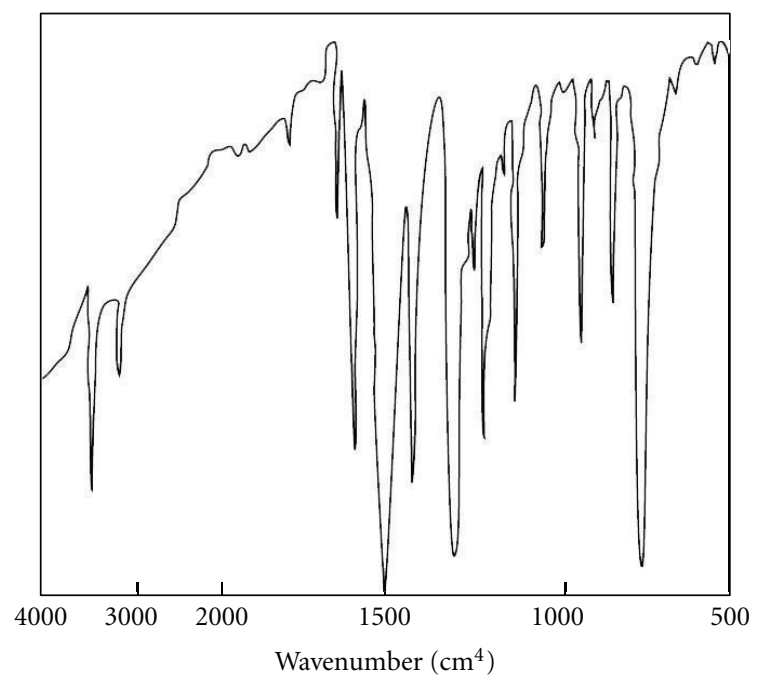

(c)

FIGURE 12: IR absorption spectra of (a) a POAP film (oxidized form), (b) ortho-aminophenol (o-AP), and (c) phenoxazine. The POAP film was prepared on BPG [16].

at $1645 \mathrm{~cm}^{-1}$ in the POAP spectrum was assigned to the stretching of the $\mathrm{C}=\mathrm{N}$ bonds present in a ladder polymer with phenoxazine rings. The absorption peaks at $1050 \mathrm{~cm}^{-1}$ and $1235 \mathrm{~cm}^{-1}$, which are characteristics of the C-O-C stretching vibration, were observed for POAP and phenoxazine, but not for the $o$-AP. Peaks at 760, 850, and $935 \mathrm{~cm}^{-1}$ for POAP were assigned to 1,2-disubstituted, 1,2,4-trisubstituted, and/or 1,2,4,5 tetrasubstituted benzene structures, respectively. All these structures were considered to be possible for POAP. Furthermore, from the fact that the absorption peak, which is assigned to the stretching vibration of the $\mathrm{C}=\mathrm{O}$ bonds of aromatic keto groups, was observed at $1670 \mathrm{~cm}^{-1}$, not only the partially hydrolyzed structure shown in Figure 7 was proposed for POAP in [16] but also the structure shown in Figure 8, which was assumed to proceed from a polymerization via $\mathrm{C}-\mathrm{N}=\mathrm{C}$ bonds, was proposed as possible structure of POAP.

The role of the laccase on the electrochemical synthesis of POAP from 0.1 M phosphate buffer ( $\mathrm{pH} 5.5$ ) solutions is described in [23]. Also, it is demonstrated in [23] that laccase can be utilized as a polymerization initiator with no need of electrochemical monomer oxidation. Raman spectra of POAP electrodeposited at $\mathrm{pH} 5.5$ with and without the presence of laccase in the polymerization bath and the spectrum of the POAP obtained by the enzyme polymerization of $o$-AP were compared in [23]. Raman bands of 2,2'dihydroxyazobenzene $(\mathrm{DHAB})$ and 3-aminophenoxazone (APZ) were observed in the spectra obtained at $\mathrm{pH}$ 5.5. Raman spectra of DHAB are characterized by the strong band at $1390-1400 \mathrm{~cm}^{-1}$ due to $\mathrm{N}=\mathrm{N}$ stretching mode. The electrochemically synthesized POAP film shows a strong band at $1397 \mathrm{~cm}^{-1}$ which was attributed to $\mathrm{N}=\mathrm{N}$ stretching mode of DHBA. This band is weaker in the spectrum of the POAP film electrodeposited in the presence of laccase and it disappears completely in the spectrum of POAP obtained by enzymatic polymerization of $o$-AP. This effect was attributed to the fact that $\mathrm{N}-\mathrm{N}$ coupling only occurs during the electrochemical oxidation of $o$-AP. Several APZ modes were also observed in the spectra of the three POAP samples (578, $\left.1150,1278,1475,1505,1605,1660 \mathrm{~cm}^{-1}\right)$. Slight shifts in 
band positions were attributed to further oxidative polymerization of APZ to POAP. Since the POAP structure contains conjugated double bonds, shifts of aromatic ring modes were considered probable. Despite the presence of laccase in the electrodeposited POAP films was confirmed by a test using syringaldazine, its contribution to Raman spectra seems to be very weak. Only weak features at 468 and $1264 \mathrm{~cm}^{-1}$ were assigned to the enzyme. The spectrum of POAP enzymatically synthesized shows a quite strong band at $1504 \mathrm{~cm}^{-1}$, whose frequency is identical to aromatic ring mode of APZ. As this frequency also coincides with the primary amine deformation mode of $o-\mathrm{AP}$, it was suggested that it is related to either monomer that did not react or APZ or short oligomers. Typical UV-Visible spectra of POAP electrodeposited in the presence and in the absence of laccase are also shown in [23]. Both spectra are characterized by a broad absorption band with maximum at $445 \mathrm{~nm}$ and $430 \mathrm{~nm}$ for the film obtained in the presence and absence of laccase, respectively. As similar bands were observed for potentiostatic $(410 \mathrm{~nm})$ [19] and potentiodynamically $(440 \mathrm{~nm})$ [39, 40] electrodeposited POAP films, the bands at $445 \mathrm{~nm}$ and $430 \mathrm{~nm}$ in [23] were attributed to conjugated $\pi$ bonds of POAP. The small difference in the position of the bands was related to possible differences in the chain lengths of POAP prepared in the presence and the absence of laccase.

With the aim to discern between two possibilities: either 2-aminophenoxazin-3-one (APZ) is the repetitive unit of POAP films, or it is incorporated onto the film structure during its synthesis, a spectroscopic characterization of soluble products in an electrolyzed $o$-AP solution was carried out in [14]. Drastic optical changes were noted after applying a constant potential of $0.85 \mathrm{~V}$ (versus RHE) on a Pt wire immersed into a spectrophotometer cell containing an $o$-AP solution. Each deconvoluted UV-Vis spectrum of this solution led to two or three main absorption bands, depending on the time at which the measurement was taken. For the purpose of comparison, the spectra of the electrolyzed solution were compared with that of APZ. While the electrolyzed solution presents an absorption band at $400 \mathrm{~nm}$, a well-defined band at $460-470 \mathrm{~nm}$ characterized APZ. The absorption band at $400 \mathrm{~nm}$ was assigned to quinone intermediates continuously formed during electrolysis (benzoquinone, benzoquinonemonoimine, benzoquinone-diimine). Although isolation of intermediates failed in [14], 2-aminophenoxazin-3-one (APZ) was identified as the final product after extraction from the electrolyzed $o$-AP solution. This assignment was supported by not only UV-Visible spectroscopy but also IR and ${ }^{1} \mathrm{H}-\mathrm{NMR}$ spectroscopy and elemental analysis. In order to establish if APZ undergoes any polymerization process, Pt and GC electrodes were cycled in a medium containing chemically synthesized APZ. While no process of charge transfer was obtained for Pt electrodes cycled in the APZ medium, GC electrodes present one well-resolved redox process. However, the electroactive response of GC was only associated to an activation process of the electrode surface after prolonged cycling. Then, voltammetric results obtained in [14] seem to indicate that APZ does not undergo any polymerization process. In another experiment carried out in [14], chemically prepared APZ was dissolved in acetone and dropped on Pt and GC electrodes in order to prepare APZ films. In this case, a single well-defined redox process was observed on the voltammograms on both Pt and GC electrodes. Due to similarities between redox responses of POAP-modified Pt and GC electrodes and APZ-modified Pt and GC electrodes, an infrared study was also performed in [14] to establish either APZ is the repetitive unit of POAP films, or it is incorporated into the film structure, or POAP films and modified electrodes cycled in an APZ medium may present similar redox responses. Then, the IR spectra of the chemically synthesized APZ, the soluble product extracted after electrolysis of $o$-AP solution, a POAP film and phenoxazine were compared in [14] (Figures 13(a), 13(b), 13(c), and 13(d), resp.). Similar IR signals for the extracted product and APZ indicated that they are the same materials. In this regard, Figures 13(a) and 13(b) present similar signals (the main coincident peaks were marked with pots) at 3300 $3500 \mathrm{~cm}^{-1}$ due to the presence of $\mathrm{NH}_{2}$ groups, and about $1600 \mathrm{~cm}^{-1}$ due to the axial stretching of the $\mathrm{C}=\mathrm{O}$ groups in the APZ structure. However, POAP presents a different spectrum Figure 13(c), and then different structures were proposed in [14] for POAP films and APZ. Common peaks are observed in Figures 13(c) and 13(d) at 1070 and $1111 \mathrm{~cm}^{-1}$, respectively, which were assigned of the stretching of the $\mathrm{C}-\mathrm{O}-\mathrm{C}$ linkages. Also, the peaks in the region $1400-1600 \mathrm{~cm}^{-1}$ were attributed to the stretching of $\mathrm{C}-\mathrm{H}$ and $\mathrm{C}-\mathrm{C}$ groups. Then, similarities were found between the POAP film spectrum and that of phenoxazine. It was concluded in [14] that, despite POAP films may present phenoxazine units and then similar redox responses can be expected for POAP films and APZ-modified electrodes, APZ does not polymerize.

Salavagione et al. [24] studied the oxidation of $o$-aminophenol, and the polymer formed, by in-situ FTIR spectroscopy. The aim of this work was to prove that most probable structure of the polymer formed in the oxidation of $o$-aminophenol contains the phenoxazine unit. Figure 14 shows the spectra of a $1 \mathrm{M} \mathrm{HClO}_{4}+5 \times 10^{-3} \mathrm{M} o$-AP solution, where a polycrystalline platinum electrode immersed in it, was polarized at different potential values. The electrode was immersed at $0.1 \mathrm{~V}$ (RHE) into the spectroelectrochemical cell and then the potential was stepped up to $0.4 \mathrm{~V}$ and the reference spectrum was collected. The potential was then polarized to higher values to oxidize the $o$-AP and the sample spectra were acquired at $0.8 \mathrm{~V}$ and $1.0 \mathrm{~V}$. In the spectrum at $0.8 \mathrm{~V}$ (RHE) (Figure 14(a)), two positive bands at 1510 and $1471 \mathrm{~cm}^{-1}$ were observed. These two bands were assigned to the aromatic $\mathrm{C}=\mathrm{C}$ stretching vibration and ring $\mathrm{C}=\mathrm{C}$ vibration of meta-disubstituted benzenes. The positive character of these bands was attributed to consumption of the species related to these features at the sample potential. At $1.0 \mathrm{~V}$ (Figure 14(b)), these two bands were observed together with a strong band at $2345 \mathrm{~cm}^{-1}$ corresponding to the formation of $\mathrm{CO}_{2}$ in the solution. No clear bands were observed at lower sample potentials. When the same spectrum is collected in deuterated water (Figure 14(c)), two negative bands at 1683 and $1645 \mathrm{~cm}^{-1}$ were observed. These bands were associated with the $\mathrm{C}=\mathrm{O}$ and $\mathrm{C}=\mathrm{N}$ stretching vibrations, respectively. Figures $15(\mathrm{a})$ and $15(\mathrm{~b})$ show the 


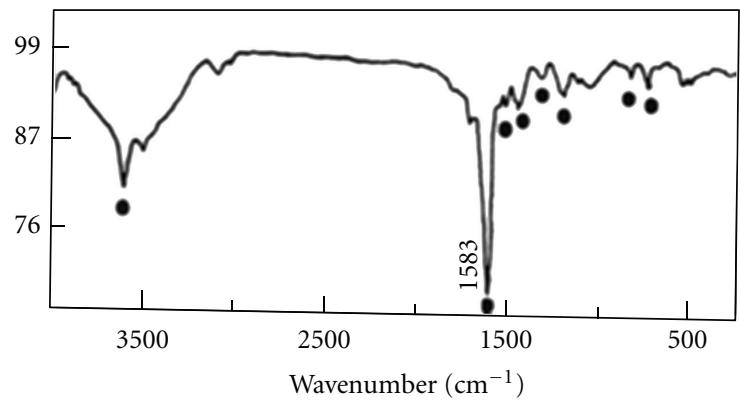

(a)

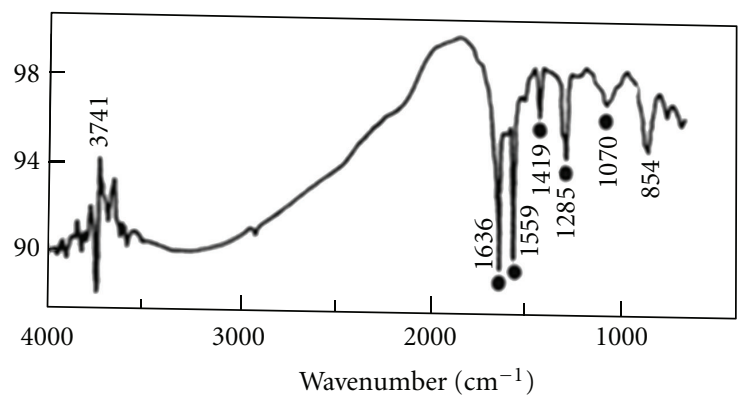

(c)

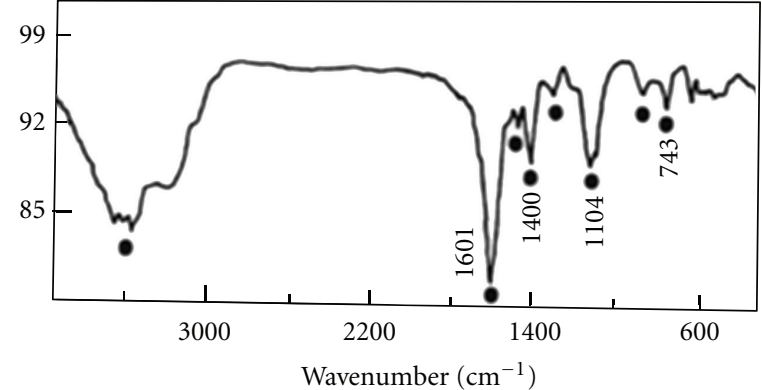

(b)

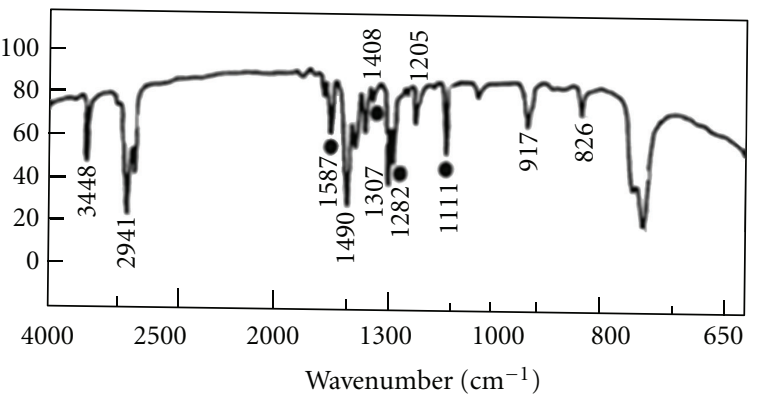

(d)

FIGURE 13: Infrared spectra of (a) extracted product from electrolyzed o-AP solution, (b) chemically synthesized 2-aminophenoxazin-3-one (APZ), (c) POAP film (prepared on Pt by cyclic voltammetry, 400 cycles), and (d) model compound (phenoxazine) [14].

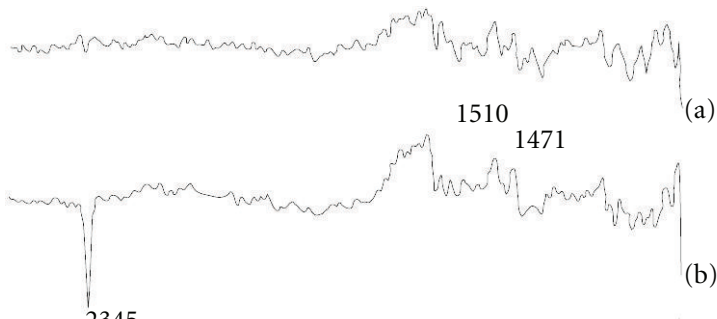

(b)

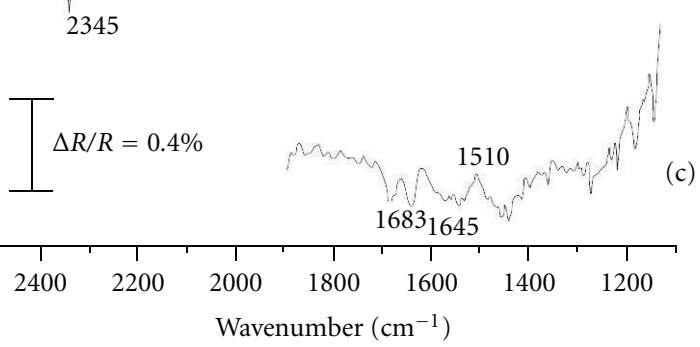

FIgURE 14: FTIR spectra for a Pt electrode in a $1 \mathrm{M} \mathrm{HClO}_{4}+$ $5 \times 10^{-3} \mathrm{M} o$-AP solution. (a) Sample potential: $0.8 \mathrm{~V}$, (b) sample potential $1.0 \mathrm{~V}$, (c) spectrum obtained at $0.9 \mathrm{~V}$ in deuterated water. P-polarized light, 100 interferograms. Reference potential: $0.4 \mathrm{~V}$ [24].

spectra of POAP in $1 \mathrm{M} \mathrm{HClO}_{4}$ solution in the absence of $o$-AP, in water and deuterated water, respectively. The reference spectrum was obtained at $0.1 \mathrm{~V}$, so it contains the vibrational information corresponding to the reduced form of the film. The electrode was then polarized to $0.7 \mathrm{~V}$ (RHE), and the sample spectrum was collected. Similar bands were observed in both spectra. Figure 15(a) displays two clear positive bands at 1513 and $1278 \mathrm{~cm}^{-1}$, and a broad negative band at $1580 \mathrm{~cm}^{-1}$. The band at $1513 \mathrm{~cm}^{-1}$ is also present in the spectra in $\mathrm{D}_{2} \mathrm{O}$ (Figure $15(\mathrm{~b})$ ) but in this case it was observed at $1517 \mathrm{~cm}^{-1}$ and was assigned to the $\mathrm{C}=\mathrm{C}$ stretching of the aromatic ring. This band was not observed after polymer oxidation. The broad negative band at $1580 \mathrm{~cm}^{-1}$ was also present in deuterated water but in this case, the band has contributions from several bands at 1564, 1606 , and $1648 \mathrm{~cm}^{-1}$, and it was assigned to a quinoid ring or $\mathrm{C}=\mathrm{N}$ stretching vibration in the phenoxazine units produced upon complete polymer oxidation (Figure 3). The $1648 \mathrm{~cm}^{-1}$ band was attributed to $\mathrm{C}=\mathrm{N}$ stretching where conjugation with phenyl group shifts its frequency to higher values and it was more clearly observed in deuterated water due to the reduced interference of water absorptions. Another negative band was observed at $1330 \mathrm{~cm}^{-1}$ in both spectra, which was also clearly seen at low potentials. This band was assigned to $\mathrm{C}=\mathrm{N}$ stretching of quinoid rings containing $\mathrm{C}=\mathrm{N}$ and $\mathrm{C}-\mathrm{N}$ groups. In order to check these assignations, the spectra for phenoxazine in the same range of potentials were also obtained in [24]. Figure 15(c) shows the spectrum obtained from a $1 \mathrm{M} \mathrm{HClO}_{4}+5 \times 10^{-4}$ phenoxazine solution in deuterated water, where the polycrystalline platinum electrode immersed in it was polarized at different potential values. A series of reference and sample spectra were collected at 0.2 and $0.7 \mathrm{~V}$ and then coadded. The spectrum obtained showed a sharp and negative band at $1508 \mathrm{~cm}^{-1}$ corresponding to the disappearance of the aromatic ring at the sample potential. A positive band at $1374 \mathrm{~cm}^{-1}$ was also observed that was assigned to the $\mathrm{C}-\mathrm{N}$ stretching of the secondary aromatic amine that also disappeared at $0.7 \mathrm{~V}$. Two negative 


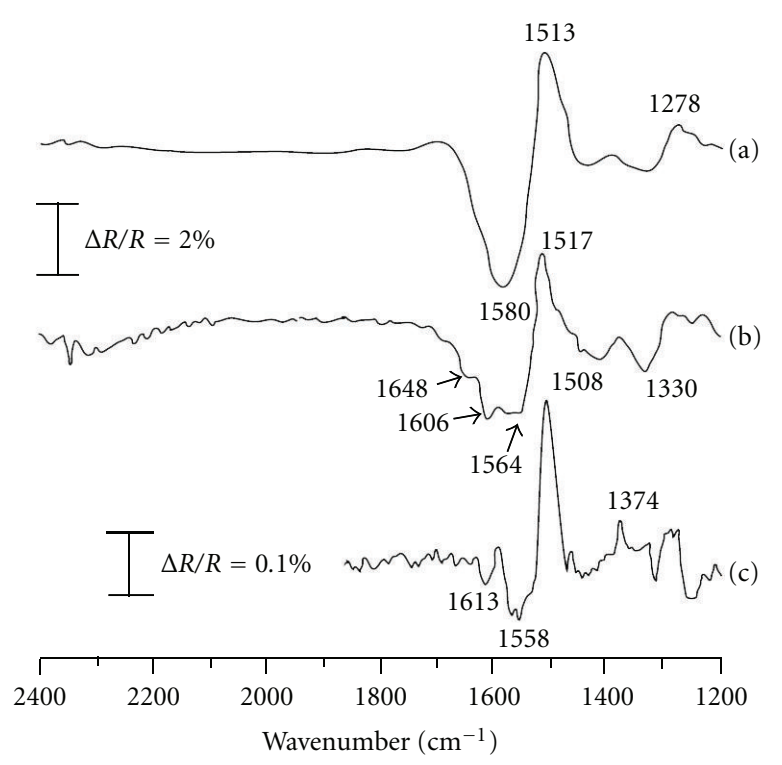

Figure 15: FTIR spectra for a Pt electrode covered with POAP in $1 \mathrm{M} \mathrm{HClO}_{4}$ solution in (a) water, (b) deuterated water. Sample potential: $0.7 \mathrm{~V}$ and reference potential: $0.1 \mathrm{~V}$. P-polarized light, 100 interferograms. (c) FTIR spectrum obtained for a Pt electrode in $1 \mathrm{M} \mathrm{HClO}_{4}+5 \times 10^{-4}$ phenoxazine solution in deuterated water. Sample potential: $0.7 \mathrm{~V}$ and reference potential: $0.4 \mathrm{~V}$. P-polarized light, 100 interferograms [24].

bands were observed at $1558 \mathrm{~cm}^{-1}$ that were associated to the $\mathrm{C}=\mathrm{N}$ stretching vibration of the imine group that is produced at higher potentials in the oxidation of phenoxazine. It was concluded in [24] that most probable structure of the polymer formed in the oxidation of $o$-aminophenol contains the phenoxazine unit as the main constituent of its structure. Theoretical calculations carried out in [24] seem to confirm that the polymer obtained by electrooxidation of $o$-AP has a ladder structure built in by phenoxazine units. In this regard, electronic density of $o$-aminophenol was calculated in [24] by employing the semiempirical self-consistent field method (AMI). It was found that $o$-aminophenol has a high electron density in the para position with respect to $-\mathrm{NH}_{2}$ group. Therefore, dimers could be formed through attack of the cation radical at that position (Section 3 ). The dimer of $o$-aminophenol has a higher electron density in the para position with respect to $-\mathrm{OH}$ group allowing closing of the phenoxazine ring.

3.2. The Redox Process of POAP in Acid Medium Studied by Spectroscopic Methods: Intermediate Species during the Redox Switching of POAP. Raman spectroscopy and voltammetry were coupled to identify structural changes during the redox process of POAP [41]. Voltammetric measurements at different perchloric acid concentrations carried out in [41] revealed the existence of two redox processes for POAP films. Raman spectra of a POAP film deposited on an Au electrode at different electrode potentials were obtained. The different bands of POAP extracted from a situ Raman spectrum acquired at $0.1 \mathrm{~V}$ are listed in Table 2. Bands at 1593, 1474,
TABLE 2: Vibration modes observed by Raman spectroscopy in POAP modified Au electrodes at $0.1 \mathrm{~V}$ in $1 \mathrm{M} \mathrm{HClO}_{4}[41]$.

\begin{tabular}{ll}
\hline Wavenumber $/ \mathrm{cm}^{-1}$ & Vibration modes \\
\hline 1638 & $-\mathrm{C}=\mathrm{N}-$ stretching of quinonimine units \\
1593 & $>\mathrm{C}=\mathrm{C}<$ stretching of quinoid units or $\mathrm{N}-\mathrm{H}^{+}$ \\
& deformation vibration on secondary amines \\
1520 & $-\mathrm{C}=\mathrm{C}-$ stretching in the aromatic ring \\
1474 & $-\mathrm{C}=\mathrm{N}-$ stretching of quinoid units \\
1390 & $\mathrm{C}-\mathrm{C}$ stretching of quinoid units \\
1328 & $>\mathrm{C}-\mathrm{N}^{\bullet+}-$ stretching \\
1160 & $\mathrm{C}-\mathrm{H}$ bending in plane \\
925 & Perchlorate vibration band \\
576 & Ring deformation of benzeniod units \\
\hline
\end{tabular}

1390 , and $1160 \mathrm{~cm}^{-1}$ were associated with quinoid groups, while bands at 1520 and $576 \mathrm{~cm}^{-1}$ were assigned to aromatic rings. The band at $1638 \mathrm{~cm}^{-1}$ was attributed to $-\mathrm{C}=\mathrm{N}-$ in quinonimine units. The intensity of some of these bands was found to be dependent on the applied potential. The behavior of the bands with the applied potential shows that when the potential increases the band at $1474 \mathrm{~cm}^{-1}$ increases and the band at $1638 \mathrm{~cm}^{-1}$ also increases until a potential about $0.2 \mathrm{~V}$ and, thereafter, it diminishes. The fitting of both bands by Lorentz curves allowed to quantify the evolution with the potential of the corresponding species associated to these bands. The integrated intensity was related with the concentrations of species responsible for these bands. The behavior of the band at $1638 \mathrm{~cm}^{-1}$ was attributed to a typical intermediate species. Since POAP has a conductivity maximum at about $0.04 \mathrm{~V}$ (SCE), the intermediate species was related to the polymer conductivity and then, it was assigned to a charged species. The existence of intermediate species was associated to an oxidation process that occurs through two consecutive reactions from the totally reduced phenoxazine form to the completely oxidized one, through a charged species, which was considered to be a cation radical. The behavior of the integrated Raman intensity of the band at $1638 \mathrm{~cm}^{-1}$ was considered to be similar to that of the band at $750 \mathrm{~nm}$ observed in the absorbance versus potential dependence in the UV-Vis region reported in [42]. As the maximum of absorbance of both bands $(750 \mathrm{~nm} \mathrm{[42]} \mathrm{and}$ $1638 \mathrm{~cm}^{-1}$ ) appear approximately at the same potential, this fact was considered to be indicative of the existence of two redox processes in the oxidation of POAP. Thus, Raman [41] and UV-Vis [42] measurements seem to suggest that the third species could be a cation radical. A redox mechanism of POAP was proposed in [41] where the first step involves mainly the anion exchange, whereas in the second step the insertion/expulsion of protons is produced.

Evidence about the existence of cation radical species during the redox conversion of POAP was also reported by Ortega [12]. Ortega studied the conducting potential range of POAP by employing cyclic voltammetry and electron spin resonance (ESR) measurements. POAP films deposited on a Pt electrode were introduced into a solution at $\mathrm{pH}$ 0.9, which was free of monomer, and then ESR spectra were recorded at 


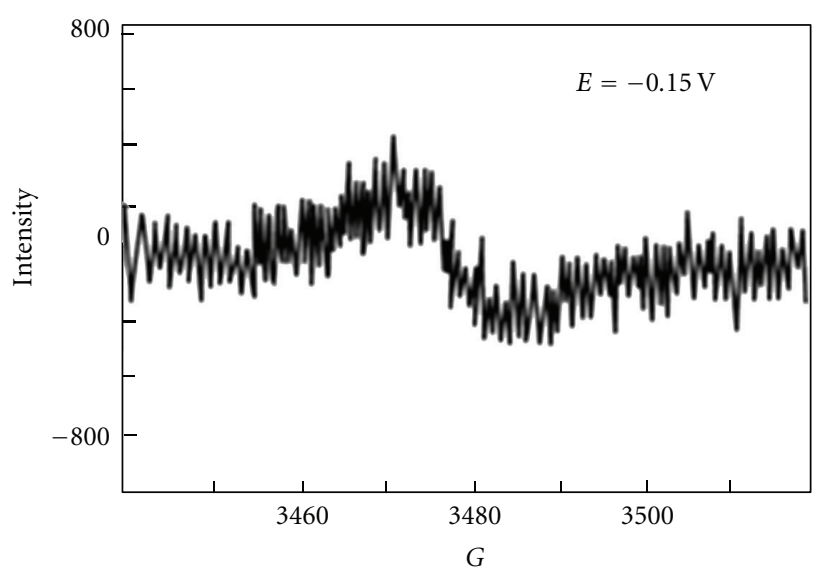

(a)

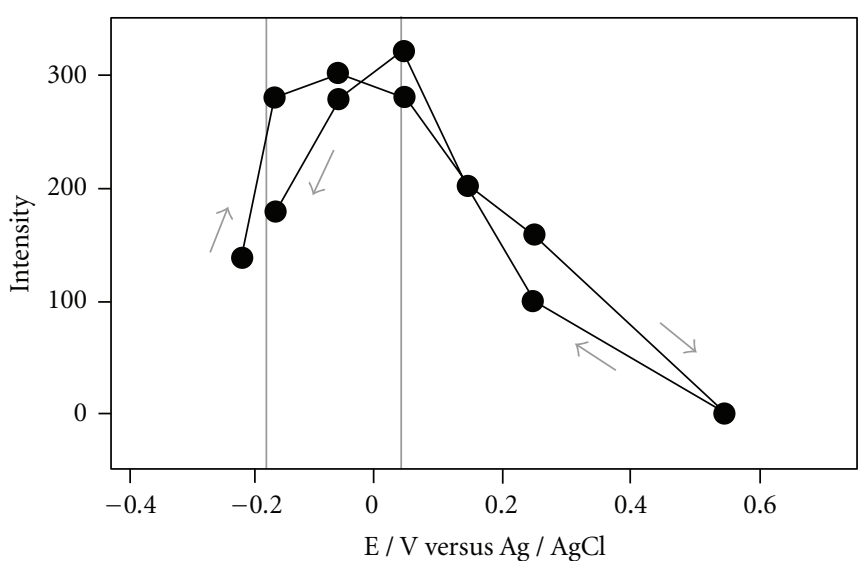

(b)

Figure 16: (a) Typical ESR spectrum of an $80 \mathrm{~nm}$ thick POAP film on Pt electrode at pH 0.9. Plot (b) illustrates the change in the ESR intensities as a function of potential. The maximum intensities are shown between the dotted straight lines [12].

TABLE 3: Vibrational bands in Raman spectra $(\lambda o=647.1 \mathrm{~nm})$ for a POAP film in a $0.5 \mathrm{M} \mathrm{H}_{2} \mathrm{SO}_{4}$ solution [19].

\begin{tabular}{ll}
\hline Wavenumber $/ \mathrm{cm}^{-1}$ & Description \\
\hline 1645 & $\mathrm{C}=\mathrm{N}$ stretching of quinonimine units \\
1598 & $\mathrm{C}=\mathrm{C}$ stretching of quinoid units \\
1472 & $\mathrm{C}=\mathrm{C}$ stretching in the aromatic ring \\
1402 & $\mathrm{C}=\mathrm{N}$ stretching of quinoid units \\
1330 & $\mathrm{C}-\mathrm{N}^{\bullet+}$ stretching of radical semiquinone \\
1170 & $\mathrm{C}-\mathrm{N}^{\bullet+}$ stretching \\
982 and 1050 & $\mathrm{C}-\mathrm{H}$ bending in plane \\
578 & Sulfate modes \\
\hline
\end{tabular}

different potentials, scanning forwards and backwards from -0.250 to $0.55 \mathrm{~V}(\mathrm{Ag} / \mathrm{AgCl})$. Figure 16 shows a typical signal at negative potentials, which starts decreasing until it reaches a very small value at $0.55 \mathrm{~V}$. The maximum in ESR spectra occurs in the potential ranging from -0.24 to approximately $0.0 \mathrm{~V}$ (SCE). The decrease and further absence of a detectable ESR signal at higher potentials than $0.55 \mathrm{~V}$ was attributed to a combination of radicals to give rise to dication species, which are not ESR active because of their paired spin. Ortega concludes that, at high positive potential values, the creation of bipolarons by a combination of polarons is possible at POAP films.

The existence of charged intermediate species together with structural changes during the redox process of POAP was also reported by Shah and Holze [19]. Shah and Holze investigated the potentiostatic electrochemical polymerization of $o$-AP at different electrode potentials by employing in-situ Raman spectroscopy. In-situ Raman spectra in the potential range, comprised between the reduced $(E=-0.2 \mathrm{~V}$ versus SCE) and the oxidized state ( $E=0.5 \mathrm{~V}$ versus SCE) of POAP in $0.5 \mathrm{M} \mathrm{H}_{2} \mathrm{SO}_{4}$ solution, are compared in [19] for both potentiostatically and potentiodynamically synthesized
POAP films. The various bands together with their possible assignments are shown in Table 3. The bands at 1598, 1472 , and $1170 \mathrm{~cm}^{-1}$ were associated to quinoid groups whereas the bands at 1522 and $578 \mathrm{~cm}^{-1}$ were attributed to benzoid rings. The band at $1330 \mathrm{~cm}^{-1}$ was assigned to semiquinone species with an intermediate structure between amines $-\mathrm{C}-\mathrm{NH}-$ and imines $-\mathrm{C}=\mathrm{N}-$ resulting in polarons. However, it was also observed in [19] an additional band at $1402 \mathrm{~cm}^{-1}$ that was completely missing in the spectra reported in [41]. It was assigned to the radical semiquinone $\mathrm{C}-\mathrm{N}^{+}$formed during the partial oxidation of $\mathrm{N}, \mathrm{N}^{\prime}$-diphenyl-1,4-phenylenediamine. The bands located at 982 and $1050 \mathrm{~cm}^{-1}$ were assigned to internal modes of the sulphate anion of the electrolyte solution. It was observed in [19] that the intensity of some of the bands depends on the applied potential. For instance, the band located at $1170 \mathrm{~cm}^{-1}$ grew in intensity when shifting the potential up to $E=0.2 \mathrm{~V}$, beyond this value it diminished with further increase of potential. The dependence of the intensity of this band on electrode potential was considered to be a characteristic feature of the oxidized form of POAP and was attributed to the $\mathrm{CH}$ bending vibrational mode of the quinoid-like rings formed during electrooxidation. The bands in the frequency range $1300-1400 \mathrm{~cm}^{-1}$ were associated mainly with the stretching vibrations of charged $\mathrm{C} \sim \mathrm{N}^{+}$segments ( $\sim$ denotes the bond intermediate between the single and double bonds). Although the Raman features of the POAP films synthesised in [19] potentiostatically and potentiodynamically were similar, marked differences in respect to the potential dependence of some bands were observed. A difference was observed particularly with respect to the potential dependence of the band around $1645 \mathrm{~cm}^{-1}$. The intensity of this band sharply increased at $0.3 \mathrm{~V}$ and slowly decreased at more positive potentials for the POAP film synthesized potentiostatically. However, in the case of POAP potentiodynamically prepared this band attained the intensity maximum at about $E=0.2 \mathrm{~V}$. The band located at $1645 \mathrm{~cm}^{-1}$ was assigned in [19] to $-\mathrm{C}=\mathrm{N}-$ in quinonimine units and was considered to 
correspond to the $\mathrm{C}-\mathrm{N}-\mathrm{C}$ bond of a heterocyclic six-member ring structure arising from ortho-coupling rather than paracoupling during the electropolymerization, resulting in a ladder polymer. As this band did not disappear even at the highest applied potential, the oxidation of fully reduced POAP synthesized potentiostatically to fully oxidized POAP was assumed to proceed via an intermediate half-oxidized state. This assumption is supported by the fact that the maximum intensity of the band at $1645 \mathrm{~cm}^{-1}$ was observed at roughly middle potential between the two redox processes observed on the cyclic voltammograms of POAP shown in [19]. The effect was associated to the maximum of polaron concentration in the polymer. The increase of potential beyond the maximum intensity, which leads to the fully oxidized state of the polymer, was attributed to a lowering in the polaron concentration probably by coupling into bipolarons. Respect to the band observed around $1598 \mathrm{~cm}^{-1}$, assigned to quinoid units, grew in intensity with increasing the electrode potential. The band at $1472 \mathrm{~cm}^{-1}$ was only observed at electrode potentials within the range $0.3 \mathrm{~V}<E<$ $0.4 \mathrm{~V}$.

Structural changes during the redox process of POAP chemically synthesized were also analyzed by employing $e x-$ situ and in-situ UV-Visible measurements. POAP was chemically synthesized by treatment of an acid solution of $o$-AP with $\mathrm{CuCl}_{2}$ and the oxidative polymerization was followed by UV-Vis spectroscopy [17]. Prior the addition of $\mathrm{CuCl}_{2}$, two absorption peaks were found on the monomer solution spectra at 258 and $460 \mathrm{~nm}$ that were assigned to the $\pi \rightarrow \pi^{*}$ transition of the aromatic structure (benzene structure) and the oxidized form of $o$-AP, respectively. A new absorption peak at $410 \mathrm{~nm}$ was developed after the addition of $\mathrm{Cu}(\mathrm{II})$ and its intensity increased with time at the expense of the peak at $460 \mathrm{~nm}$. The $410 \mathrm{~nm}$ peak was assigned to the radical cation (oxidized form) of POAP. The solid polymer synthesized in [17] was also examined by X-ray Photoelectron Spectroscopy (XPS) in [17]. The authors remarked that the C1s, N1s, and O1s spectral features were similar to those reported for POAP electrochemically prepared in other works [44]. The carbon spectrum was deconvoluted to estimate the extent of carbon involved in $\mathrm{C}-\mathrm{C}, \mathrm{C}-\mathrm{N}$, and $\mathrm{C}=\mathrm{O}$ bonds. A ratio of $3: 2: 1$, indicating a good degree of polymerization, was obtained in [17]. It was concluded in [17] that the polymer growth proceeds mainly through the reactions between the growing polymer (POAP) and oxidized monomer units and subsequent cyclization of the functional units in the polymer leads to a ladder structure. The relative reflectance change $\Delta R / R$ as a function of the wavelength within the visible spectrum region was also employed in [17] to study the structure of electrochemically synthesized POAP films. The wavelength dependence of the relative reflectance $\Delta R / R$ for a POAP film electrochemically deposited on $\mathrm{Pt}$ when it is polarized at various potentials is shown in [17]. A broad absorption band extending from $410 \mathrm{~nm}$ to $532 \mathrm{~nm}$ was observed for polarization within the range $0.1 \mathrm{~V}-1.1 \mathrm{~V}$ (SCE). The band intensity increased with increasing the potential, turning the film dark brown. In addition, a slight blue shift, simultaneously with the intensity increase, was noted. The absorption band was assigned to the formation of radical cations at POAP, as the polymer matrix becomes oxidized. The blue shift with the increase of the potential was attributed to a larger fraction of radical cation species contained in the polymer as it becomes more oxidized. According to the authors of [17], the oxidation of POAP is not complete even at $0.5 \mathrm{~V}-0.6 \mathrm{~V}$ (SCE), then, the broad band at $410 \mathrm{~nm}$ was considered to be composed by $\pi \rightarrow \pi^{*}$ transitions proceeding from both the oxidized (quinonoid) and reduced (N-phenyl-p-phenylenediamine) units (Figure 9).

UV-V is spectra of POAP films synthesized potentiostatically at different electrode potentials were analyzed by Shah and Holtze [19]. Figures 17(a) and 17(b) show UV-Vis spectra of a POAP film at different electrode potentials within the range $-0.2 \mathrm{~V}<E<0.6 \mathrm{~V}$ (SCE). Three absorption peaks located at $\lambda=350,410$, and $610 \mathrm{~nm}$ are observed, respectively. At $E=-0.2 \mathrm{~V}$, the polymer is in its reduced state and the corresponding spectra shows an absorption band located at approximately at $\lambda=350 \mathrm{~nm}$. This band was attributed to the phenoxazine structure. With increasing the potential, oxidation of POAP takes place, leading then to the formation of radical cations. It was observed that as the potential is increased from $-0.2 \mathrm{~V}$ to $0.1 \mathrm{~V}$, the intensity of the band at band at $\lambda=350 \mathrm{~nm}$ decreases and also at $0.1 \mathrm{~V}$ it is split into at least two bands. With further increase of the potential the intensity of one of these bands is diminished, while the other one starts to shift to lower energies and changes into a broad maximum around $\lambda=410 \mathrm{~nm}$. The absorption band at $\lambda=610 \mathrm{~nm}$ increases in intensity up to $0.2 \mathrm{~V}$ and then it becomes nearly constant with further increase of potential. The behavior of the in-situ UV-Vis spectra of POAP films potentiostatically synthesized presented in [19] was considered as a verification of previous results reported by Ortega in [12] indicating that the redox transition of POAP from its completely reduced state to its completely oxidized state proceeds though two consecutive reactions in which a charged intermediate species takes part.

A spectroelectrochemical study of the redox process of POAP is reported in [42]. Absorbance changes in the wavelength region comprised between $300 \mathrm{~nm}$ and $800 \mathrm{~nm}$, at different $\mathrm{pHs}$ values and in the presence of different supporting electrolytes, were recorded and analyzed at different degrees of oxidation of POAP. Two types of experiments were carried out in [42]: (i) at a fixed wavelength, the electrode potential was swept at scan rates comprised between 0.005 and $0.03 \mathrm{~V}$ $\mathrm{s}^{-1}$ and (ii) at a fixed electrode potential, the wavelength was scanned. Figure 18 shows the spectra of POAP films at different electrode potentials in the region where the film is electrochemically active $(-0.2 \mathrm{~V}<E<0.7 \mathrm{~V}$ versus SCE). The absorbance of the film at the negative potential limit $(-0.2 \mathrm{~V})$ was attributed to the tail of the UV band related to the $\pi \rightarrow \pi^{*}$ transition of the basic aromatic structure of the phenoxazine units. A reaction scheme for the POAP redox switching, which also includes protonation reactions, is shown in [42]. The redox switching of POAP was interpreted in terms of the oxidation of amine groups to imine groups. As POAP is progressively oxidized, several changes in the spectral response were observed in [42]: (i) a decrease of the absorbance in the wavelength region about $340 \mathrm{~nm}$; 


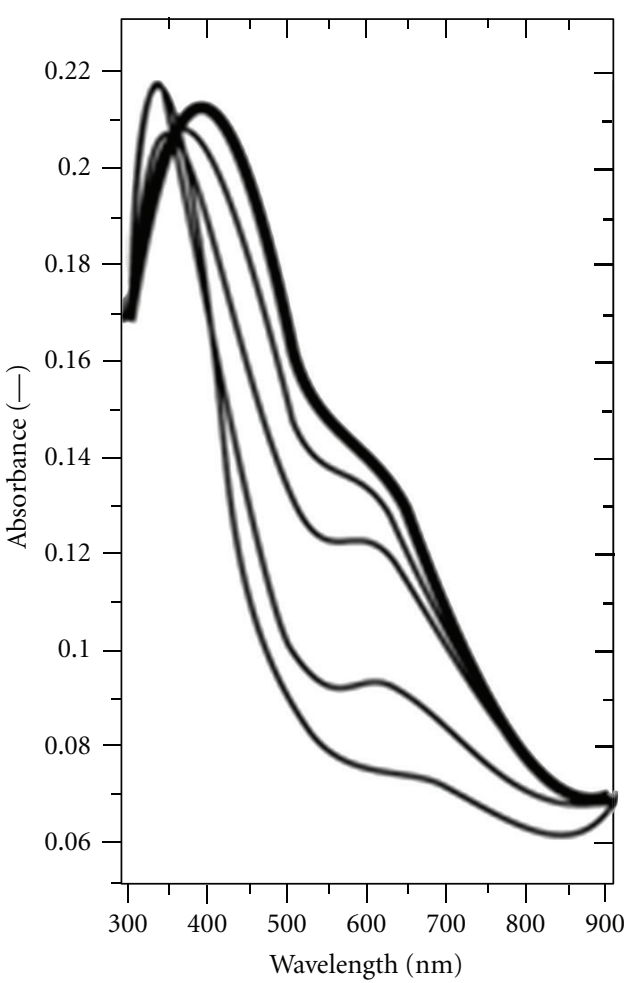

(a)

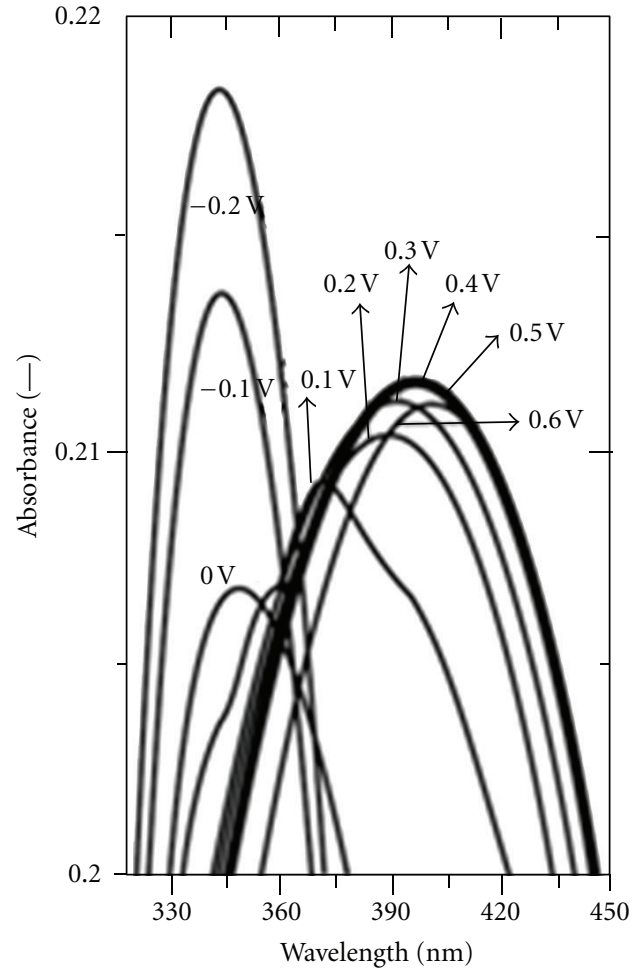

(b)

FIGURe 17: (a) In-situ UV-Vis spectra of POAP at different applied electrode potentials. The POAP film was prepared on ITO coated glass at $0.8 \mathrm{~V}$ ( $1 \mathrm{~h}$ electrolysis) from a solution containing $0.05 \mathrm{M} o$-AP in $0.5 \mathrm{M} \mathrm{H}_{2} \mathrm{SO}_{4}$ solution. (b) Enlarged spectra of (a) between 320 and $450 \mathrm{~nm}$ [19].

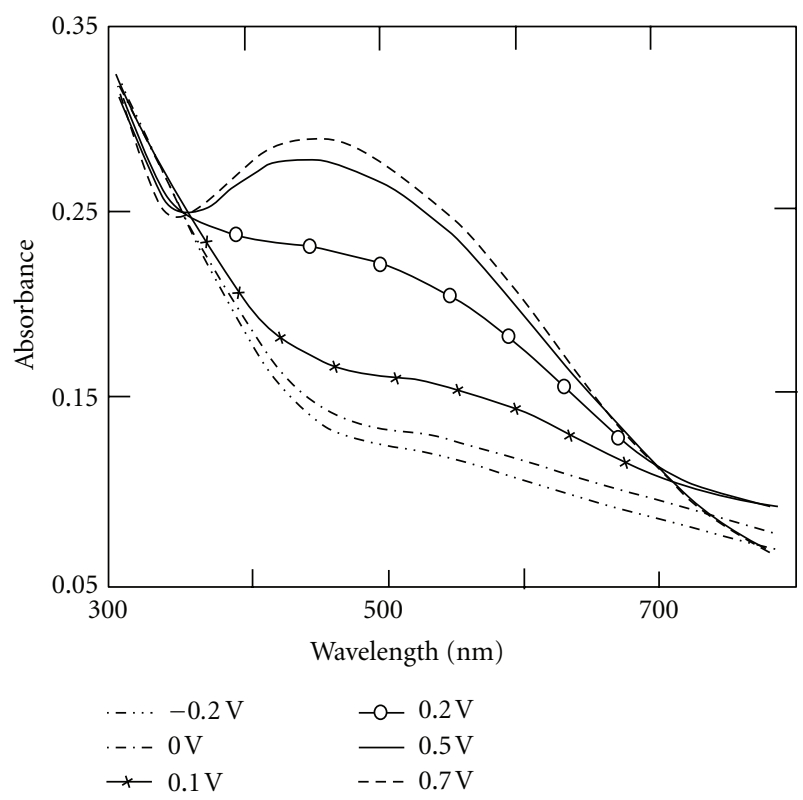

FIGURE 18: Absorbance as a function of wavelength at fixed electrode potentials, $E$, in $0.4 \mathrm{M} \mathrm{NaClO}_{4}+0.1 \mathrm{M} \mathrm{HClO}_{4}(-\cdots-)-0.2$ $\left.\mathrm{V},(---\longrightarrow) 0.0 \mathrm{~V},(-\mathrm{x}-) 0.1 \mathrm{~V},(-\mathrm{O}-) 0.2 \mathrm{~V},(-) 0.5 \mathrm{~V},(--)^{-}\right) 0.7 \mathrm{~V}$ [42].

(ii) a broad maximum was developed at $450 \mathrm{~nm}$; (iii) in the region of $\lambda>750 \mathrm{~nm}$, the absorbance first increases with potential up to $E=0.1 \mathrm{~V}$ and then, for $E>0.1 \mathrm{~V}$, the absorbance decreases. The broad band growing at $450 \mathrm{~nm}$ as the potential increases in the positive direction was assigned to the partially oxidized phenoxazine structure. While the decreases of the absorbance at $\lambda=340 \mathrm{~nm}$, as the potential increases in the positive direction, was attributed to the disappearance of the reduced form of POAP, the increase of the absorbance at $\lambda=450 \mathrm{~nm}$ was associated to the in-crease of the oxidized form with the potential scan. At $\lambda=750 \mathrm{~nm}$, the absorbance presents a maximum at $E=0.15 \mathrm{~V}$, which was considered to be indicative of the existence of a transient species. Spectra of reduced $(E=-0.2 \mathrm{~V})$ and oxidized $(E=0.7 \mathrm{~V})$ states of POAP were also found to be dependent on $\mathrm{pH}$. The absorbance difference $\Delta A=A_{\mathrm{ox}}-A_{\text {red }}$ was analyzed in [42] and represented, at different pHs as a function of the wavelength while the polymer goes from the reduced state to the oxidized one. A slight increase of the UV tail was observed under these conditions and it was attributed to a poor protonation of the polymer as it becomes oxidized. This effect was associated to the conversion of one protonated amino group to a nonprotonated imino group. It was concluded in [42] that as the $\mathrm{pH}$ increases the reduction of the oxidized species becomes progressively hindered and consequently the amount of oxidable species available in the film decreases. Experiments carried out in [42] in the presence of different anions (perchlorate, sulphate, and benzenesulphonate) at $\mathrm{pH} 1$ showed that the absorbance with 


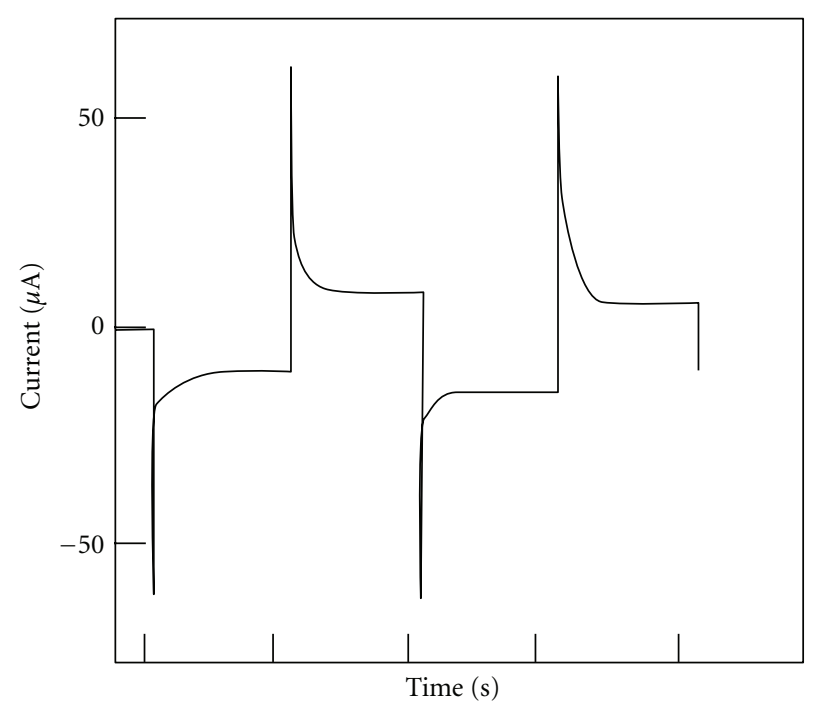

(a)

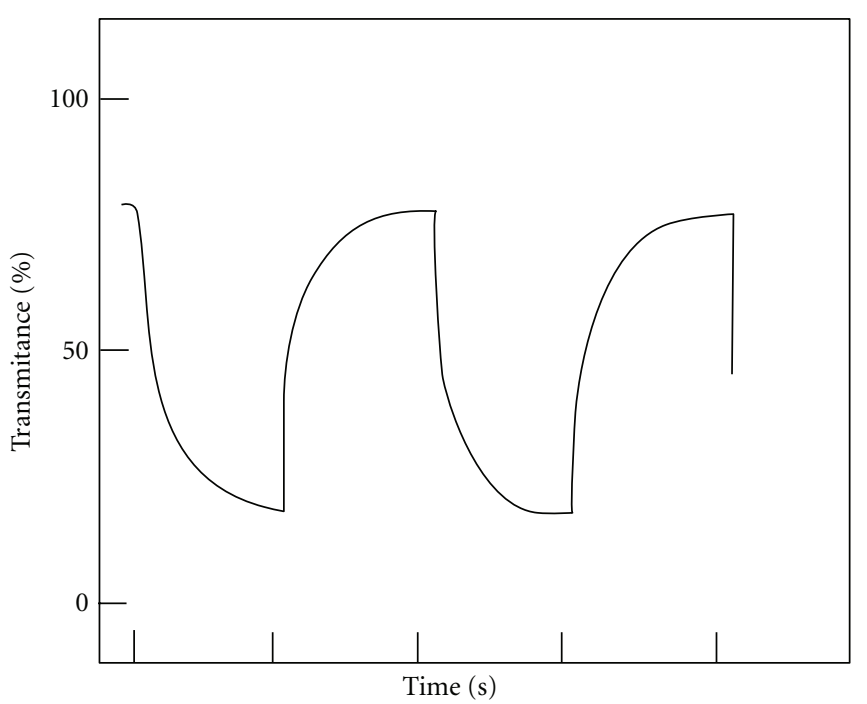

(b)

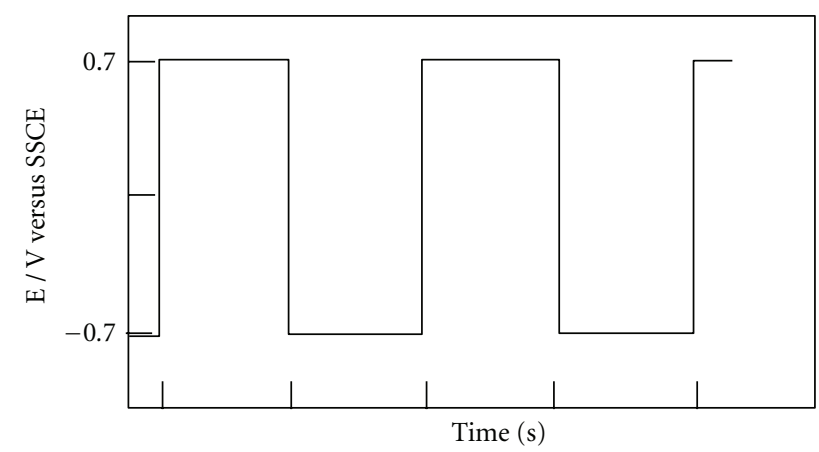

(c)

FIGURE 19: The responses of current and transmittance at $440 \mathrm{~nm}$ of the POAP film- $(\phi=0.2 \mu \mathrm{m})$ coated ITO electrode to a potential pulse between $-0.7 \mathrm{~V}$ and $0.7 \mathrm{~V}$ in $0.2 \mathrm{M} \mathrm{NaClO}_{4}$ aqueous solution $(\mathrm{pH} 1)$. Electrode area, $0.20 \mathrm{~cm}^{2}[16]$.

potential at $440 \mathrm{~nm}$ decreases in the sequence $\mathrm{ClO}_{4}{ }^{-}>$ $\mathrm{HSO}_{4}{ }^{-}>$benzenesulphonate. That is, the absorbance decreases with increasing the size of the anion. The transient response at $750 \mathrm{~nm}$ was also be dependent on the nature of the anion of the supporting electrolyte, thus, for benzenesulphonate the change was smaller but the decay was slower than for perchlorate and bisulphate.

The UV-Vis absorption spectra of the oxidized and reduced forms of a POAP film-coated ITO electrode were analyzed by Kunimura and coworkers [16]. The maximum absorption peak of the oxidized form was observed at $400 \mathrm{~nm}$ and that of the reduced one at $600 \mathrm{~nm}$. A reversible colour change of the film between brown and pale green was observed when the electrode potential was cycled between $-0.7 \mathrm{~V}$ and $0.7 \mathrm{~V}$ versus SCE. Figure 19 shows the response of current and transmittance at $440 \mathrm{~nm}$ of the electrode during the potential step within the electrochromic region. The colour accumulated in the anodic step was bleached in the cathodic one. No substantial change in the transmittance response of the electrode was observed, even after the potential stepping of 100 cycles. The brown colour of the oxidized form remained substantially unchanged even after standing it in air at open circuit for a period of 6 months. On the other hand, the reduced form was gradually oxidized by oxygen in air and consequently became coloured. The colourlessness of the reduced form was held under an atmosphere of nitrogen gas.

Barbero et al. [45] reported a spectrophotometric and ellipsometric study of POAP formed by electropolymerization of $o$-AP on gold and platinum surfaces. POAP films of different thickness $(d)$ were synthesized by CV in [45]. The films were subjected to different polarization conditions (reduced and oxidized states) and the ellipsometric readings of the polarizer and analyzer ( $\Delta$ and $\Psi$ values, resp.) at $\lambda=$ $541.6 \mathrm{~nm}$ were obtained. It was observed that up to one particular film thickness $(50 \mathrm{~nm})$, reversing the potential from the oxidized to the reduced state and vice versa, resulted in the same ellipsometric readings for each state (i.e., oxidized and reduced), thus showing that the film behaves reversibly from the optical viewpoint. Experimental values of $\Psi$ and $\Delta$ for both, oxidized and the reduced states, for the different thicknesses at one wavelength, were fitted by a single film model in which it was assumed that the complex refractive index of the film $(\tilde{n})$ for each state (oxidized $\left(\tilde{n}_{\mathrm{ox}}\right)$ or reduced 
$\left(\tilde{n}_{\text {red }}\right)$ ) was independent of the film thickness. The best fit allowed determining $\tilde{n}$ and $k$ (real part of the complex refractive index) common to all thicknesses for each state. The corresponding $\tilde{n}$ values resulted: $\widetilde{n}_{\text {red }}=1.704-i 0.02$ and $\widetilde{n}_{\text {ox }}$ $=1.699-i 0.175$ at $\lambda=546.1 \mathrm{~nm}$. These values indicate that the film is transparent in the reduced state and opaque in the oxidized one. As the value of the real part of $\widetilde{n}$ results common to both states and it is slightly higher than that reported for PANI, the authors of [45] infer that the structure of POAP is similar to that proposed for PANI. Also, from the $\Psi$ versus $\Delta$ dependence, it was concluded that POAP in the oxidized state should have a higher thickness $\left(d_{\mathrm{ox}}\right)$ than in the reduced $\left(d_{r}\right)$ one. From the poor fitting at $d>$ $50 \mathrm{~nm}$, it was suggested that thick POAP films exhibit a less densely packed structure or an increasing surface roughness, or both of them, as compared with thin POAP films. Similar experiments as those above described were carried out in [45] at different wavelengths within the range $350 \mathrm{~nm}<$ $\lambda<650 \mathrm{~nm}$. In this regard, by repeating the same fitting procedure, the $n$ and $k$ values together with the thickness were determined for both film conditions (oxidized and reduced states). It was observed that for low thicknesses, $d_{\mathrm{ox}}$ is bigger than $d_{r}$. However, this difference tends to disappears for high film thicknesses. A voltammetric cathodic charge versus ellipsometric thickness relationship for POAP is also reported in [45]. It was observed that the charge, $Q$, during the negative potential scan increases linearly with the film thickness obtained by ellipsometry. However, as $d$ increased beyond $40 \mathrm{~nm}$, the slope of the $Q$ versus $d$ dependence became smaller. This fact was considered to be indicative of a decrease in the film density, that is, the ellipsometric thickness would be bigger than the electrochemically active portion of the film.

3.3. Spectroscopic Studies of POAP Films Synthesized in Basic and Neutral Media. Jackowska and coworkers [43] studied the chemical and electrochemical oxidation of $o$-AP over a wide $\mathrm{pH}$ range. A mixture of at least eight different compounds obtained from the chemical oxidation of $o$-AP were separated chromatographically in [43] and then their surface enhanced Raman scattering (SERS) spectra were compared. 3-APZ (3-aminophenoxazone) was identified as the main product by recording an IR spectrum and the second product of the chemical oxidation of $o$-AP was identified as 22 'dihydroxyazobenzene (DHAB). $3 \mathrm{APZ}$ is formed by the simultaneous $\mathrm{N}-\mathrm{C}$ and $\mathrm{O}-\mathrm{C}$ coupling of $o$-AP monomer units. The N-N coupling yields DHAB. With regard to the solution $\mathrm{pH}$, while $\mathrm{DHAB}$ is formed mainly in neutral and basic solutions of $o$-AP, at low $\mathrm{pH}$ values, $3 \mathrm{APZ}$ was considered to be the main oxidation product. Also, the two main products identified in [43] after electrochemical oxidation of $o$-AP were $3 \mathrm{APZ}$ and $\mathrm{DHAB}$. $3 \mathrm{APZ}$ could be oxidized at the electrode forming a ladder structure polymer.

Figure 20 shows SERS spectra of a silver electrode immersed in $o-\mathrm{AP}$ at different $\mathrm{pH}$ and electrode potential values. A band at $1395 \mathrm{~cm}^{-1}$ is clearly observed in al spectra. This band was assigned to nitrosophenol. As can be seen from Figure 20, while this band exhibits a strong intensity in alkaline solutions, it is weaker in the acidic medium. By comparing the behavior of this band with the band at $1390 \mathrm{~cm}^{-1}$ observed in the SERS spectra of nitrosobenzene, it was concluded that at open-circuit potential, $o$-AP on the silver electrode is already oxidized into two major products: 3 -APZ and DHAB. The SERS spectrum of the $o$-AP solution also depends on the applied electrode potential. At pH 3.0 (Figure 20(a)) the spectrum is most intense at the stationary potential electrode $E=0.01 \mathrm{~V}$, weak at $-0.2 \mathrm{~V}$, and almost absent at $-0.4 \mathrm{~V}$ after the reduction of 3-APZ. At $\mathrm{pH} 7.5$ (Figure 20(b)), the overall intensity of the spectrum significantly diminishes at more negative potential values (about $-0.6 \mathrm{~V}$ ). In alkaline solutions (Figure 20(c)), the main band ascribed to 3-APZ and DHAB disappear at $-0.7 \mathrm{~V}$ after a reduction of azo compounds to hydrazo compounds. The spectrum observed at more negative potential values in neutral and alkaline $\mathrm{pHs}$ was attributed to hydrazo species, which are products of the reduction of DHAB.

The electropolymerization of $o$-AP in alkaline media $(\mathrm{pH}=12)$ on copper electrodes was studied in [22]. IR spectrometry was employed to characterize the polymer films. The IR spectrum of the POAP film and that of the 2aminophenol compound are compared in [22]. The film spectrum did not present the $\mathrm{O}-\mathrm{H}$ band $\left(3375 \mathrm{~cm}^{-1}\right.$ for the stretching vibration and $1268 \mathrm{~cm}^{-1}$ for the bonding vibration), characteristics of the 2-aminophenol. Instead of these bands, that of C-O-C at $1297.7 \mathrm{~cm}^{-1}$ was observed. This spectral difference was considered to be consistent with an electropolymerization process of $o-\mathrm{AP}$, which proceeds through the anodic oxidation of the monomer. The absence of the band at $1700 \mathrm{~cm}^{-1}$, assigned to $\mathrm{C}=\mathrm{O}$ groups, confirmed the only formation of a polyether compound. The presence of the characteristic strong absorptions, corresponding to the bands of the $-\mathrm{NH}_{2}$ group in the ranges 300$3500 \mathrm{~cm}^{-1}$ and $1590-1610 \mathrm{~cm}^{-1}$, was observed in the films IR spectra. ESCA analysis of the POAP films was also carried out in [22]. The spectrum of the oxygen 1s was interpreted as being composed of different peaks 531.5, 532.9, and $533.9 \mathrm{eV}$, attributed, respectively, to the oxygen bonding to a metal in an oxide, to the oxygen of water adsorbed molecules or hydroxide group $(\mathrm{OH})$ and to $\mathrm{C}-\mathrm{O}-\mathrm{C}$ bonds. The third component at high energy $(286.5 \mathrm{eV})$ of $\mathrm{C}_{1 \mathrm{~s}}$ spectrum was assigned to $\mathrm{C}-\mathrm{O}-\mathrm{C}$ bonds, while the lowenergy components were considered to be characteristic of $\mathrm{C}-\mathrm{C}$ bonds $(284.6 \mathrm{eV})$ and $\mathrm{C}-\mathrm{N}$ bonds $(285.5 \mathrm{eV})$. The $\mathrm{N}_{1 \mathrm{~s}}$ spectrum was assigned to the three types of bonds of the 2-aminophenol: $\mathrm{O}-\mathrm{N}, \mathrm{N}-\mathrm{H}$, and $\mathrm{C}-\mathrm{N}(389.9,400.3$, and $401.2 \mathrm{eV}$ ). Copper in its oxidized forms was also observed in the film: $\mathrm{Cu}_{2} \mathrm{O}(932.6 \mathrm{eV})$ and $\mathrm{CuO}(933.7 \mathrm{eV})$. The higher energy component $(934.5 \mathrm{eV})$ was attributed to a complex formed with the organic compounds. IR studies were also carried out in [22] and they seem to indicate that the POAP film-growing process in alkaline media involves the deprotonation of the aminophenol molecule, which is probably chemisorbed at the metal surface, followed by oxidation and electropolymerization reactions. In this whole process, the polymerization affects the $-\mathrm{OH}$ group by the formation of $\mathrm{C}-\mathrm{O}-\mathrm{C}$ bond while the $-\mathrm{NH}_{2}$ groups are preserved. 


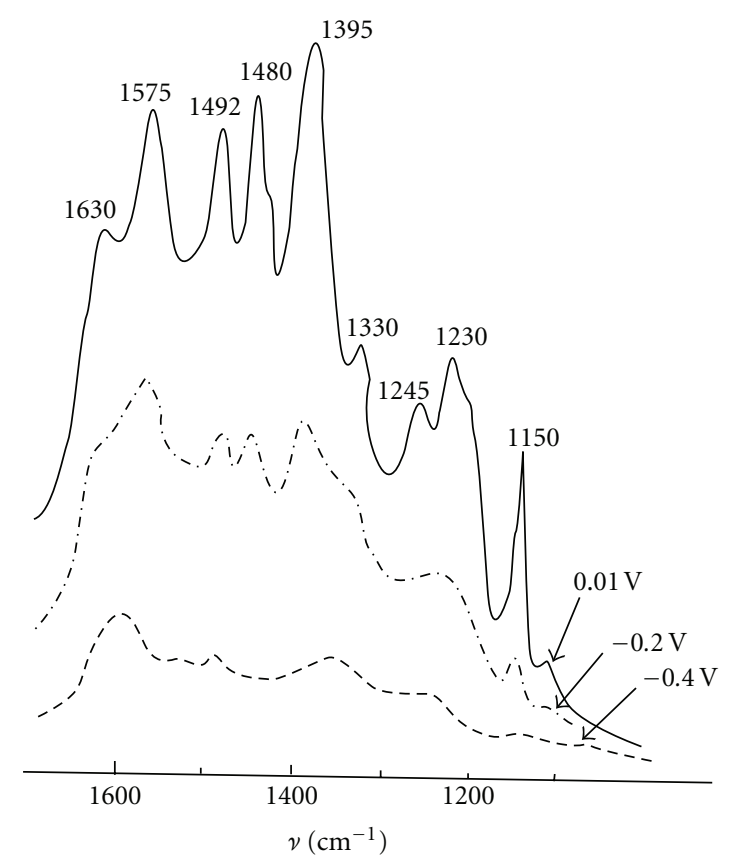

(a)

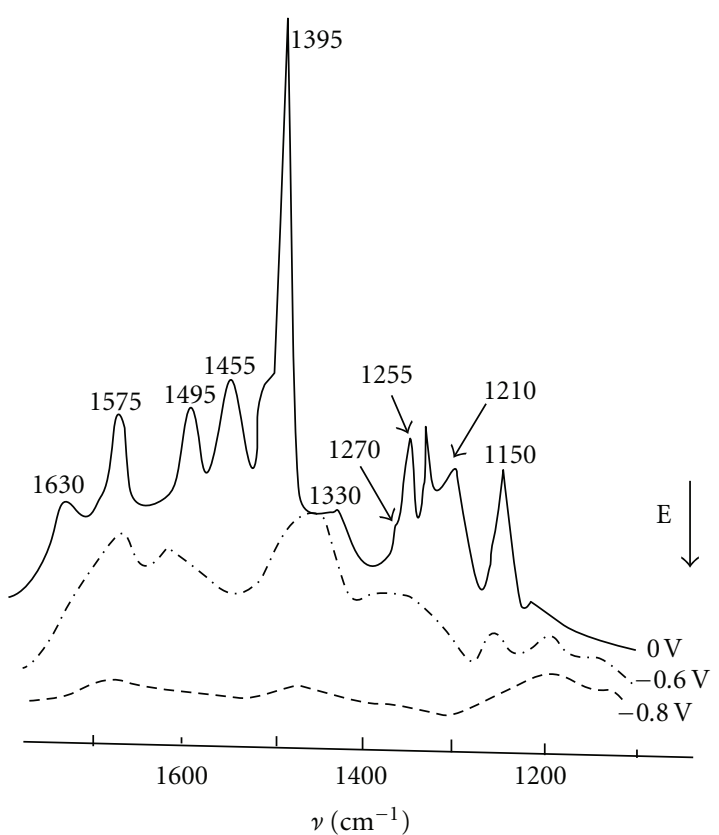

(b)

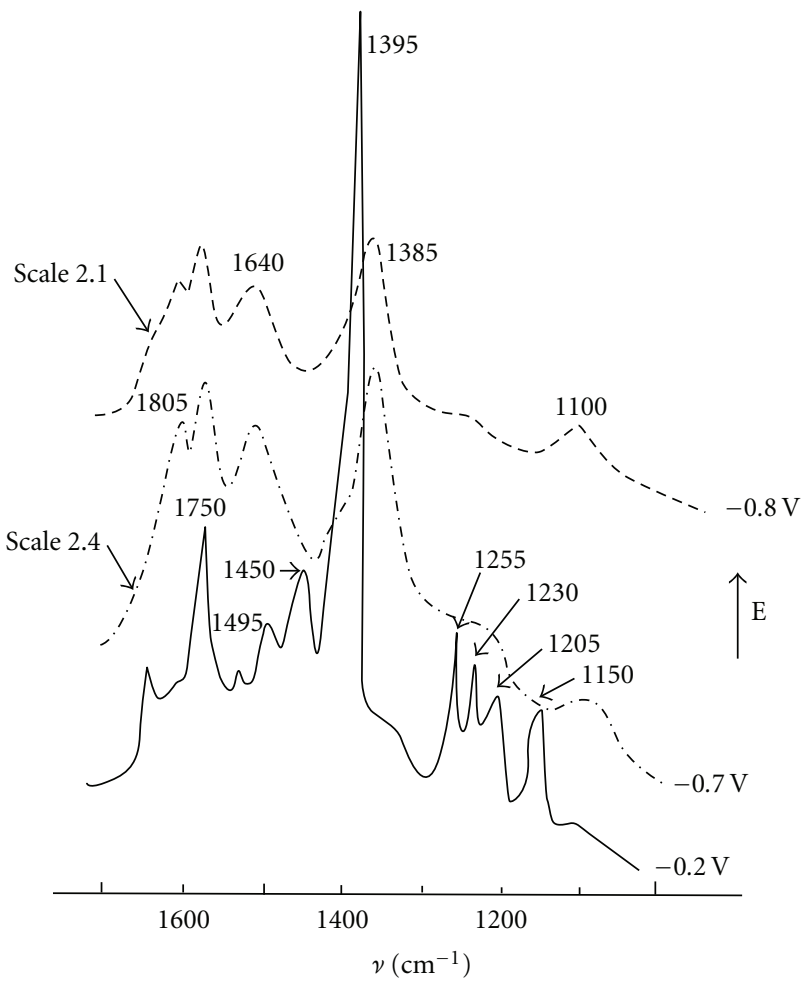

(c)

Figure 20: SERS spectra of $o$-AP solutions at (a) pH 3.0, (b) pH 7.5, and (c) pH 9.7, for different electrode potentials [43].

\section{Formation Mechanism of Poly(o-Aminophenol) (POAP) Films}

On the basis of electrochemical and spectroscopic studies reported in [10], Barbero et al. explained the formation of POAP films in acid medium employing the generalized mechanism of anodic oxidation of anilines in aqueous media solutions. These authors propose that a radical cation $o$ - $\mathrm{AP}^{+} \bullet$ is formed in a first charge transfer step and then, it may follow the reaction paths shown in Figure 21. The $o$ $\mathrm{AP}^{+}$radical may dimerize by either $\mathrm{C}-\mathrm{C}$ coupling or $\mathrm{C}-\mathrm{N}$ coupling to give species (I) and (II), respectively, (Figure 21). 


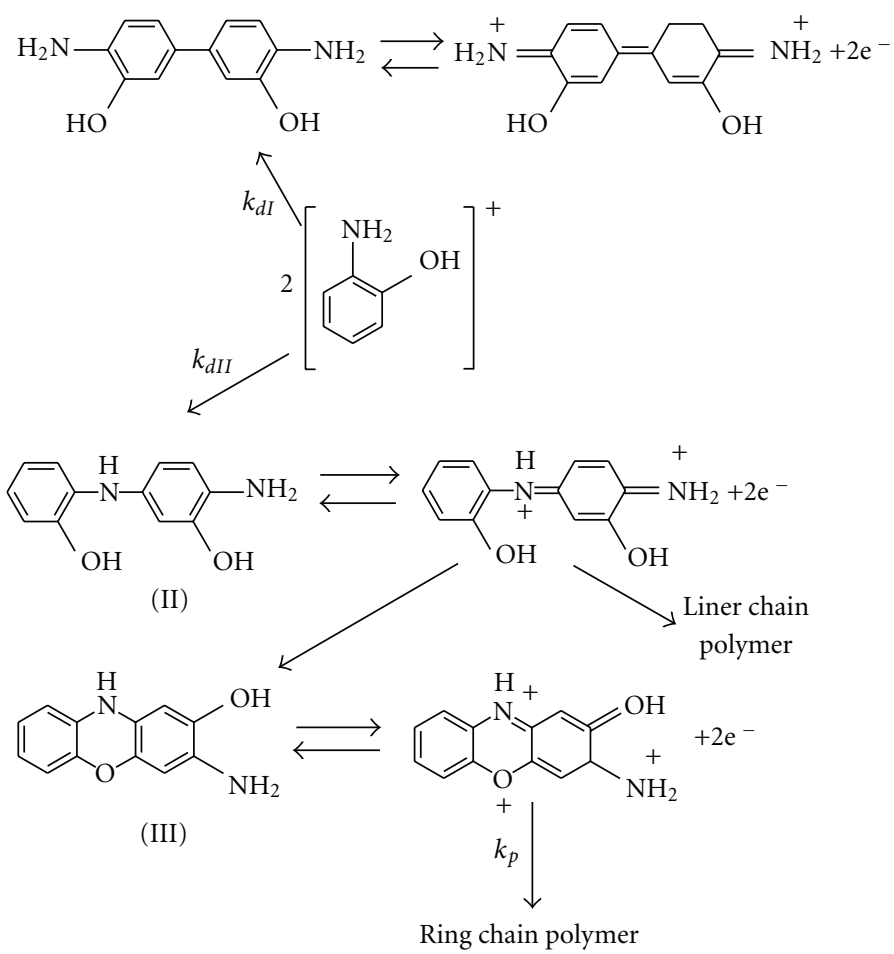

FIGURE 21: Mechanism of anodic oxidation of $o$-AP [10].

The dimmers oxidize to the corresponding dications. The oxidized dimmer II can suffer a cyclization reaction to give species III, which is oxidized further to 3APZ. The product distributions analyzed in [10] allowed the authors establishing that the rate constants for the dimers formation to follow the order $k_{d I}<k_{d I I}$ and both rate constants are higher than the cyclization rate, $\left(k_{c}\right)$ to give species III. However, the rate of polymerization $\left(k_{p}\right)$, to obtain the immobilized couple on the electrode surface resulted lower than the cyclization rate $\left(k_{c}\right)$. The possibility that the dication of compound II can polymerize was not disregarded. Then, the formation of a composite of two different films one of linear chain structure similar to PANI and another with a phenoxazine-like chain structure was assumed to be possible. The latter product was considered to be the predominant one in $[10]$.

Gonçalves and coworkers [14] postulate that electrochemical oxidation of $o$-AP consists in a first oxidation step involving a two-electron transfer to form radical cations followed by chemical couplings of radical cation-radical cation or radical-monomer species $(\mathrm{E}(\mathrm{CE}))$ mechanism to form a ladder polymer with phenoxazine units. However, radical cations can also react quickly near the electrode surface, and after the first step involving two electrons, soluble products are easily formed by hydrolysis (Figure 22). Thus, besides a film with a ladder structure, Gonçalves and coworkers propose that oxidation of $o$-AP can produce intermediate benzoquinone monoamine after successive cycling. Particularly at less controlled conditions such as at higher final potentials and lower scan rates, monoamines can react with neutral $o$-AP giving an intermediate (2-amino-o-indophenol) prior to cyclization to APZ. The low solubility of POAP films was attributed to the stiffness of the ladder phenoxazine backbone, which also seems to justify the low conductivity of POAP $\left(10^{-7} \mathrm{~s} \mathrm{~cm}^{-1}\right)$ as compared with that of PANI $\left(1 \mathrm{~s} \mathrm{~cm}^{-1}\right)$.

Jackowska and coworkers combined cyclic voltammetry and surface-enhanced Raman scattering (SERS) measurements to study the formation mechanism of POAP at different pHs $[43,46]$. SERS results reported in [46] from a roughened silver electrode in contact with an $o$-AP solution had suggested the presence of at least two oxidation products (nitrophenol and nitrosophenol) on the electrode surface at open-circuit potential. Typical cyclic voltammetric curves obtained for a silver electrode in $0.1 \mathrm{M} \mathrm{LiClO}_{4}$ solutions of $o$ $\mathrm{AP}$ at different $\mathrm{pH}$ values are shown in [46]. In all cases, one pair of redox peaks was observed. However, two $\mathrm{pH}$ ranges with different curve features could be distinguished. Within the range of moderate $\mathrm{pHs}$ (9.1 and 7.2), potentials of anodic $\left(E_{\mathrm{pa}}\right)$ and cathodic $\left(E_{\mathrm{pc}}\right)$ peaks changed slightly with $\mathrm{pH}$. However, within the second $\mathrm{pH}$ range ( $\mathrm{pH} 3.6$ and below), $E_{\mathrm{pa}}$ and $E_{\mathrm{pc}}$ were strongly $\mathrm{pH}$ dependent. These different features of the cyclic voltammetry curves within these two $\mathrm{pH}$ ranges were attributed to the presence of two different electroactive oxidation products on the surface of the silver electrode. In order to identify of products formed during the potential cycling, cyclic voltammograms were recorded in different solutions of $0.1 \mathrm{M} \mathrm{LiClO}_{4}$ : (i) saturated with nitrosobenzene $(\mathrm{pH} 10,6.7,3.6,1.3)$, (ii) saturated with azoxybenzene ( $\mathrm{pH} 10,7.0,3.7,2.0)$, and (iii) on a roughened 


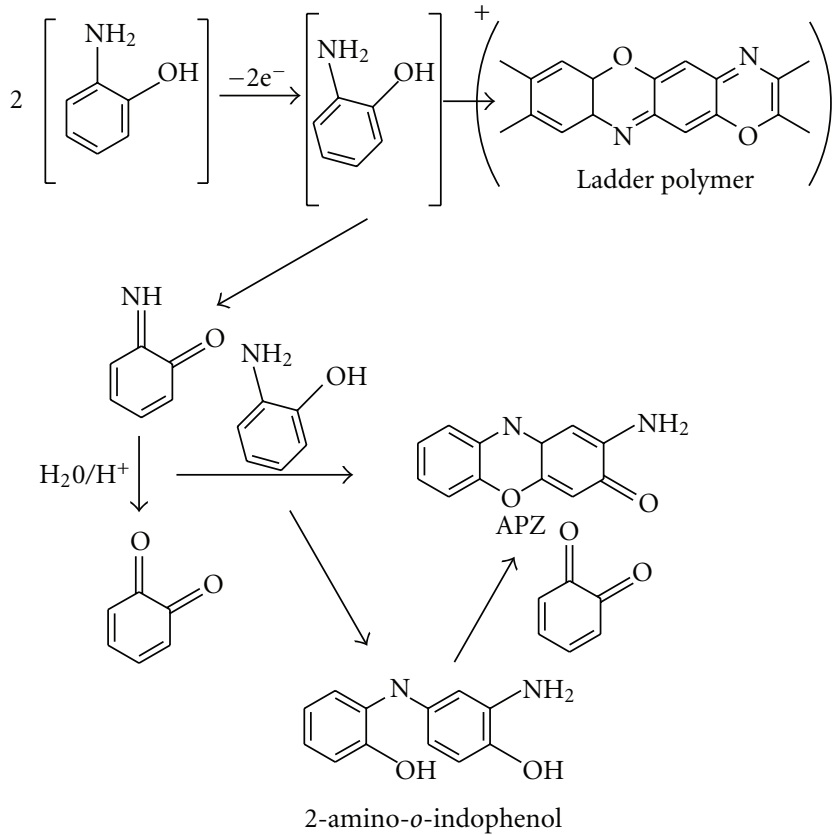

FIGURE 22: Mechanism of $o$-AP electrooxidation in aqueous acidic medium [14].

silver electrode modified with 3-APZ. In the first two cases, no redox peaks were found in the potential range from 0.15 to $-0.5 \mathrm{~V}$ (SCE) for nitrosobenzene ( $\mathrm{pH} 1.3$ ) and azoxybenzene $(\mathrm{pH}$ 2.0) solutions. However, for nitrosobenzene solutions with $\mathrm{pH}$ values in the range 10-3.6, two pairs of slightly $\mathrm{pH}$-dependent redox peaks were recorded. The first pair of redox peaks was assigned to the well-known reduction reaction of nitrosobenzene $\left(\mathrm{C}_{6} \mathrm{H}_{5} \mathrm{NO}\right)$ to phenylhydroxylamine $\left(\mathrm{C}_{6} \mathrm{H}_{5} \mathrm{NHOH}\right)$ and the reoxidation of $\mathrm{C}_{6} \mathrm{H}_{5} \mathrm{NO}$ to $\mathrm{C}_{6} \mathrm{H}_{5} \mathrm{NHOH}$. The second pair of redox peaks was attributed to the reduction of azoxy species, which are formed by the chemical reaction of $\mathrm{C}_{6} \mathrm{H}_{5} \mathrm{NO}$ and $\mathrm{C}_{6} \mathrm{H}_{5} \mathrm{NHOH}$ to hydrazobenzene and reoxidation of hydrazobenzene to azobenzene. In solutions containing azoxybenzene, only one pair of redox peaks was recorded. As it is well known that azoxybenzene can undergo irreversible reduction to azobenzene followed by a two-electron reversible reduction to hydrazobenzene, the observed pair of redox peaks was ascribed to an azo-hydrazo redox reaction. Cyclic voltammetry curves for nitrosobenzene, azoxybenzene, and $o$-AP solutions at moderate $\mathrm{pH}$ (about 7) were also compared in [43]. As a pair of redox peaks was obtained for $o$-AP solutions in neutral and alkaline media, it was attributed to the azo-hydrazo couple. Thus, Jackowska and coworkers assume that after oxidation of $o$-AP to $o-\mathrm{AP}^{\bullet+}$ in neutral and alkaline media, dimerization of $o-\mathrm{AP}^{\bullet+}$ by $\mathrm{N}-\mathrm{N}$ coupling takes place. Then, the formation of azo species from $o$-AP on the silver electrode in an alkaline medium was postulated, and the reaction path was considered to be similar to that proposed for the formation of azobenzene from the aniline molecule (Figure 23). In order to interpret the cyclic voltammetry curves for 0 -AP solutions at $\mathrm{pH}<4$, cyclic voltammograms for a roughened

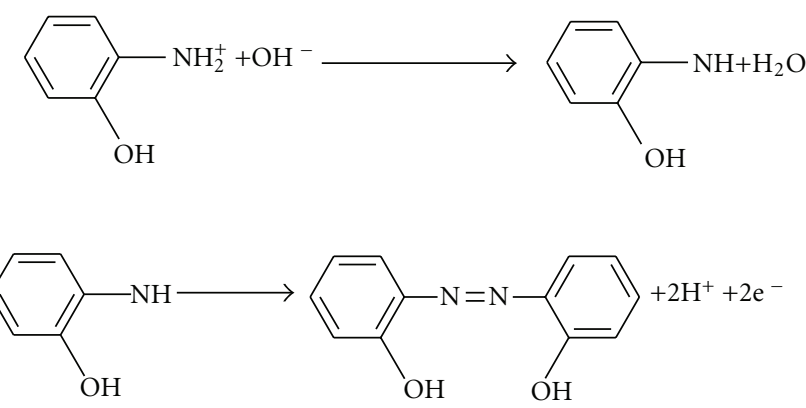

FIGURE 23: Formation of azo species from $o$-AP on the silver electrode in an alkaline medium [43].

silver electrode modified with 3-APZ (Ag/3-APZ) were analyzed in the potential range from -0.2 to $-0.4 \mathrm{~V}$ (SCE). Only one pair of reversible redox peaks was observed on the curves. The dependence of the peak potentials on $\mathrm{pH}$ indicated that protons are essential for the electrode reaction. It was not possible to determine $E_{\mathrm{pa}}$ and $E_{\mathrm{pc}}$ precisely for the $\mathrm{Ag} / 3-\mathrm{APZ}$ electrode at $\mathrm{pH}<2$ since $E_{p}$ is in the potential range of silver oxidation, but data obtained for $\mathrm{Pt} / 3-\mathrm{APZ}$ electrode at $\mathrm{pH}<3$ suggested that $E_{\mathrm{p}}$ changes at a rate about $60 \mathrm{mV} / \mathrm{pH}$. This relationship indicates that protons and electrons take part in the electrode reaction of $3-\mathrm{APZ}$ in a $1: 1$ ratio. Then, it was postulated that in more acidic solutions, the favored path after oxidation of $o$-AP to $o-\mathrm{AP}^{\bullet+}$ is dimerization of $o-\mathrm{AP}^{\bullet+}$ with $\mathrm{N}-\mathrm{C}$ coupling resulting in the cyclic dimer 3-APZ.

\section{Concluding Remarks}

POAP films have been synthesized by electrochemical oxidation of acid and basic $o$-AP solutions. While 2-2' dihydroxyazobenzene (DHAB) is formed mainly in neutral and basic solutions, at low $\mathrm{pH}$ values, 3-aminophenoxazone (3APZ) was considered to be the main electrooxidation product. $3 \mathrm{APZ}$ is formed by the simultaneous $\mathrm{N}-\mathrm{C}$ and $\mathrm{O}-\mathrm{C}$ coupling of $o$-AP monomer units and it can be oxidized at the electrode forming a ladder polymer with a phenoxazine-like chain structure. The $\mathrm{N}-\mathrm{N}$ coupling yields DHAB in basic media $[43,46]$.

On the basis of spectroscopic measurements, different structures have been proposed for POAP. Besides a completely ring-closed structure with phenoxazine units $[10$, $12,14,19]$, other two structures, a partially ring opened and another partially hydrolyzed, have been formulated for POAP. In-situ Raman spectroscopy measurements suggest that the POAP matrix contains alternating oxidized (quinonoid) and reduced (N-phenyl-p-phenylenediamine) repeating units $[17,18]$. The 1,4 -substituted molecular structure of POAP proposed by Zhang et al. [17, 18] allows explaining the interaction of the polymer with metal cations. The cation capturing process by POAP was attributed to simultaneous presence of hydroxyl and amino groups of the polymeric backbone, in which the lone-pair electrons are available to coordinate with metal cations. 
With regard to mechanisms of POAP formation, most authors $[10,12-14,19]$ postulate that in acidic solutions the favored path after oxidation of $o-\mathrm{AP}$ to $o-\mathrm{AP}^{\bullet+}$ is dimerization of $o-\mathrm{AP}^{\bullet+}$ with $\mathrm{N}-\mathrm{C}$ coupling resulting in the cyclic dimer 3-APZ. Oxidation of 3APZ leads a ladder structure polymer with phenoxazine units. However, the $o$ - $\mathrm{AP}^{+} \cdot$ radical may also dimerize by $\mathrm{C}-\mathrm{C}$ coupling. Thus, the formation of a composite of two different films, one of linear chain structure similar to polyaniline (PANI) and the other with a phenoxazine-like chain structure, was assumed to be possible for POAP. Gonçalves and coworkers [14] postulate that electrochemical oxidation of $o$-AP consists in a first oxidation step involving a two-electron transfer to form radical cations followed by chemical couplings of radical cation-radical cation or radical-monomer species to yield a ladder polymer with phenoxazine units. However, the radical cations can also react quickly near the electrode surface to produce soluble products by hydrolysis. Then, besides a film with a ladder structure, Gonçalves and coworkers propose that oxidation of $o$-AP can produce intermediate benzoquinone monoamine after successive cycling. Spectroscopic measurements demonstrate the formation of azo species from alkaline solutions of $o$-AP $[23,43]$. Jackowska and coworkers postulate that after oxidation of $o$-AP to $o-\mathrm{AP}^{\bullet+}$, dimerization of $o$ $\mathrm{AP}^{\bullet+}$ by $\mathrm{N}-\mathrm{N}$ coupling takes place and the reaction path in basic media was assumed to be similar to that proposed for the formation of azobenzene from the aniline molecule.

It was demonstrated that some substances, such as sodium dodecyl sulphate [13], increase the rate of polymerization of $o$-aminophenol in acid media because in its presence the monomer can more easily reach the electrode surface and produce more monocation radical, than in its absence. Also, some electrode surface pretreatments affect the electropolymerization rate of $o$-aminophenol. It was proved that the electron-transfer rate from a POAP film to a glassy carbon electrode is twice when the glassy carbon surface was previously electrochemically pretreated (electrode subjected to $1.85 \mathrm{~V}$ (SCE) for $5 \mathrm{~min}$ in a $0.1 \mathrm{M}$ $\mathrm{H}_{2} \mathrm{SO}_{4}$ solution), as compared with an untreated surface.

The redox switching of POAP from the totally reduced form to the completely oxidized one in acid medium was also studied by employing UV-Vis and Raman spectroscopies and ERS measurements $[12,19,41,42]$. The existence of transient species during the redox process of POAP was demonstrated analyzing the potential dependence of some spectral bands. In this regard, the redox process of POAP was interpreted in [42] in terms of the oxidation of amine groups to imine groups. To this end, absorbance changes in the wavelength region comprised between $300 \mathrm{~nm}$ and $800 \mathrm{~nm}$, at different $\mathrm{pHs}$ values, were recorded and analysed at different degrees of oxidation of POAP. Three bands were studied: $340 \mathrm{~nm}$, $450, \mathrm{~nm}$ and $750 \mathrm{~nm}$. The band at $340 \mathrm{~nm}$ was attributed to the phenoxazine structure of the polymer. The band at $450 \mathrm{~nm}$ was assigned to the partially oxidized phenoxazine structure. At $\lambda=750 \mathrm{~nm}$, the absorbance presents a maximum at $E=0.15 \mathrm{~V}$ (SCE), which was considered to be indicative of the existence of a transient species. The same conclusion was reached by studying the potential dependence of some bands of the Raman spectra of POAP [41]. For instance, the intensities of the band at $1638 \mathrm{~cm}^{-1}$, attributed to $-\mathrm{C}=\mathrm{N}-$ in quinonimine units of the band at $1474 \mathrm{~cm}^{-1}$ assigned to the $-\mathrm{C}=\mathrm{N}-$ stretching, were analyzed in [41] as functions of the applied electrode potential. It was found that when the potential increases, the band at $1474 \mathrm{~cm}^{-1}$ increases and the band at $1638 \mathrm{~cm}^{-1}$ also increases until a potential about $0.2 \mathrm{~V}$ and, thereafter, it diminishes. This behavior was considered indicative of the existence of an intermediate species. The existence of intermediate species was associated to an oxidation process that occurs through two consecutive reactions through a charged species, which was considered to be a cation radical. From another point of view, the effect was also associated to the maximum of polaron concentration in the polymer. The increase of potential beyond the maximum intensity was attributed to a lowering in the polaron concentration probably by coupling into bipolarons. In the same sense, although the Raman features of the POAP films synthesized potentiostatically and potentiodynamically were similar [19], marked differences in respect to the potential dependence of some bands were observed. A difference was observed particularly with respect to the potential dependence of the band around $1645 \mathrm{~cm}^{-1}$ assigned to the $\mathrm{C}=\mathrm{N}$ stretching of quinonimine units. The intensity of this band sharply increased at $0.3 \mathrm{~V}$ and slowly decreased at more positive potentials for POAP films synthesized potentiostatically. However, in the case of POAP films potentiodynamically prepared, this band attained the intensity maximum at about $E=0.2 \mathrm{~V}$. Thus, the oxidation of POAP synthesized potentiostatically was assumed to proceed via an intermediate half-oxidized state. The behavior of the in-situ UV-Vis spectra of POAP films potentiostatically synthesized [19] also demonstrate that the redox transition of POAP proceeds though two consecutive reactions in which a charged intermediate species takes part. Evidence about the existence of cation radical species during the redox conversion of POAP was also obtained by ERS measurements [12].

\section{Acknowledgments}

The authors gratefully acknowledge the Consejo Nacional de Investigaciones Científicas y Técnicas (CONICET) and also the Facultad de Ciencias Exactas, National University of La Plata (UNLP).

\section{References}

[1] C. Barbero, J. J. Silber, and L. Sereno, "Electrochemical properties of poly-ortho-aminophenol modified electrodes in aqueous acid solutions," Journal of Electroanalytical Chemistry, vol. 291, no. 1-2, pp. 81-101, 1990.

[2] T. Komura, Y. Ito, T. Yamaguti, and K. Takahasi, "Chargetransport processes at poly- $O$-aminophenol film electrodes: electron hopping accompanied by proton exchange," Electrochimica Acta, vol. 43, no. 7, pp. 723-731, 1997.

[3] R. I. Tucceri, "Surface resistance measurements on thin gold film electrodes coated with poly (o-aminophenol) films," Journal of Electroanalytical Chemistry, vol. 505, no. 1-2, pp. 72-84, 2001. 
[4] O. Levin, V. Kondratiev, and V. Malev, "Charge transfer processes at poly-o-phenylenediamine and poly-o-aminophenol films," Electrochimica Acta, vol. 50, no. 7-8, pp. 1573-1585, 2005.

[5] J. F. Rodriguez Nieto, R. I. Tucceri, and D. Posadas, "EIS detection of the partial oxidation of polymers derived from aryl amines," Journal of Electroanalytical Chemistry, vol. 403, no. 1-2, pp. 241-244, 1996.

[6] R. Tucceri, "The effect of high positive potentials on the different charge transport and charge transfer parameters of poly (o-amnophenol) modified electrodes. A study using cyclic voltammetry, steady-state rotating disc electrode voltammetry and AC impedance measurements," Journal of New Materials for Electrochemical Systems, vol. 8, no. 4, pp. 305-317, 2005.

[7] R. Tucceri, "A review about the surface resistance technique in electrochemistry," Surface Science Reports, vol. 56, no. 3-4, pp. 85-157, 2004.

[8] A. Bonfranceschi, A. Pérez Córdoba, S. Keunchkarian, S. Zapata, and R. Tucceri, "Transport across poly(o-aminophenol) modified electrodes in contact with media containing redox active couples. A study using rotating disc electrode voltammetry," Journal of Electroanalytical Chemistry, vol. 477, no. 1, pp. 1-13, 1999.

[9] R. Tucceri, "A review about the charge conduction process at poly(o-aminopenol) film electrodes," The Open Physical Chemistry Journal, vol. 4, pp. 62-77, 2010.

[10] C. Barbero, J. J. Silber, and L. Sereno, "Formation of a novel electroactive film by electropolymerization of ortho-aminophenol. Study of its chemical structure and formation mechanism. Electropolymerization of analogous compounds," Journal of Electroanalytical Chemistry, vol. 263, no. 2, pp. 333-352, 1989.

[11] R. Tucceri, "Practical applications of poly(o-aminopenol) film electrodes," The Open Physical Chemistry Journal, vol. 4, pp. 45-61, 2010.

[12] J. M. Ortega, "Conducting potential range for poly(o-aminophenol)," Thin Solid Films, vol. 371, no. 1, pp. 28-35, 2000.

[13] R. Ojani, J.-B. Raoof, and S. Fathi, "Poly (o-aminophenol) film prepared in the presence of sodium dodecyl sulphate: aplication for nickel-ion dispersion and the elecctrocatalytic oxidation of methanol and ethylene glycol," Electrochimica Acta, vol. 54, pp. 2190-2196, 2009.

[14] D. Gonçalves, R. C. Faria, M. Yonashiro, and L. O. S. Bulhões, "Electrochemical oxidation of $o$-aminophenol in aqueous acidic medium: formation of film and soluble products," Journal of Electroanalytical Chemistry, vol. 487, no. 2, pp. 90-99, 2000.

[15] Y. Yang and Z. Lin, "Effects of surface oxide species on the electropolymerization of $o$-aminophenol on pretreated glassy carbon electrodes," Synthetic Metals, vol. 78, no. 2, pp. 111115, 1996.

[16] S. Kunimura, T. Ohsaka, and N. Oyama, "Preparation of thin polymeric films on electrode surfaces by electropolymerization of o-aminophenol," Macromolecules, vol. 21, no. 4, pp. 894-900, 1988.

[17] A. Q. Zhang, C. Q. Cui, Y. Z. Chen, and J. Y. Lee, "Synthesis and electrochromic properties of poly-o-aminophenol," Journal of Electroanalytical Chemistry, vol. 373, no. 1-2, pp. 115121, 1994.

[18] A. Q. Zhang, C. Q. Cui, and J. Y. Lee, "Metal-polymer interactions in the $\mathrm{Ag}^{+} /$poly-o-aminophenol system," Journal of Electroanalytical Chemistry, vol. 413, no. 1-2, pp. 143-151, 1996.
[19] A. U. H. A. Shah and R. Holze, "Poly(o-aminophenol) with two redox processes: a spectroelectrochemical study," Journal of Electroanalytical Chemistry, vol. 597, no. 2, pp. 95-102, 2006.

[20] M. C. Miras, A. Badano, M. M. Bruno, and C. Barbero, "Nitric oxide electrochemical sensors based on hybrid films of conducting polymers and metal phtalocyanines," Portugaliae Electrochimica Acta, vol. 21, pp. 235-243, 2003.

[21] S. M. Golabi and A. Nozad, "Electrocatalytic oxidation of methanol at lower potentials on glassy carbon electrode modified by platinum and platinum alloys incorporated in poly $(o-$ aminophenol) film," Electroanalysis, vol. 15, no. 4, pp. 278286, 2003.

[22] A. Guenbour, A. Kacemi, A. Benbachir, and L. Aries, "Electropolymerization of 2-aminophenol. Electrochemical and spectroscopic studies," Progress in Organic Coatings, vol. 38, no. 2, pp. 121-126, 2000.

[23] B. Pałys, M. Marzec, and J. Rogalski, "Poly-o-aminophenol as a laccase mediator and influence of the enzyme on the polymer electrodeposition," Bioelectrochemistry, vol. 80, no. 1, pp. 43$48,2010$.

[24] H. J. Salavagione, J. Arias, P. Garcés, E. Morallón, C. Barbero, and J. L. Vázquez, "Spectroelectrochemical study of the oxidation of aminophenols on platinum electrode in acid medium," Journal of Electroanalytical Chemistry, vol. 565, no. 2, pp. 375383, 2004.

[25] A. Q. Zhang, Y. Z. Chen, and Z. W. Tian, "Metal-polymer interaction in the $\mathrm{Ag}^{+} /$polyaniline system," Acta PhysicoChimica Sinica, vol. 9, p. 523, 1993.

[26] J. Yano, H. Kawakami, S. Yamasaki, and Y. Kanno, "Cation Capturing Ability and the Potential Response of a Poly (o-aminophenol) Film Electrode to Dissolved Ferric Ions," Journal of the Electrochemical Society, vol. 148, no. 2, pp. E61-E65, 2001.

[27] J. Yano, H. Kawakami, and S. Yamasaki, "Potential response of poly(o-aminophenol) film electrode to dissolved ferric ion," Synthetic Metals, vol. 102, no. 1-3, p. 1335, 1999.

[28] S. Kuraşun, B. Z. Ekinci, A. Paaşahan, and E. Ekinci, "Preparation and properties of amperometric uric acid sensor based on poly(2-aminophenol)," Journal of Applied Polymer Science, vol. 120, no. 1, pp. 406-410, 2011.

[29] W. Tao, D. Pan, Y. Liu, L. Nie, and S. Yao, "An amperometric hydrogen peroxide sensor based on immobilization of hemoglobin in poly $(o$-aminophenol $)$ film at iron-cobalt hexacyanoferrate-modified gold electrode," Analytical Biochemistry, vol. 338, no. 2, pp. 332-340, 2005.

[30] W. Tao, Y. Liu, D. Pan, L. Nie, and S. Yao, "Study on the enhancement of catalytic activity for hemoglobin by quinhydrone in poly(o-aminophenol) film," Bioelectrochemistry, vol. 65, no. 1, pp. 51-58, 2004.

[31] D. Pan, J. Chen, L. Nie, W. Tao, and S. Yao, "Amperometric glucose biosensor based on immobilization of glucose oxidase in electropolymerized $o$-aminophenol film at Prussian bluemodified platinum electrode," Electrochimica Acta, vol. 49, no. 5, pp. 795-801, 2004.

[32] E. Miland, A. J. Miranda Ordieres, P. Tuñón Blanco, M. R. Smyth, and C. Ó. Fágáin, "Poly(o-aminophenol)-modified bienzyme carbon paste electrode for the detection of uric acid," Talanta, vol. 43, no. 5, pp. 785-796, 1996.

[33] M. J. Lobo-Castañón, A. J. Miranda-Ordieres, and P. TuñónBlanco, "A bienzyme-poly-(o-phenylenediamine)-modified carbon paste electrode for the amperometric detection of Llactate," Analytica Chimica Acta, vol. 346, no. 2, pp. 165-174, 1997. 
[34] M. J. Lobo, A. J. Miranda, J. M. López-Fonseca, and P. Tuñón, "Electrocatalytic detection of nicotinamide coenzymes by poly (o-aminophenol) — and poly(o-phenylenediamine)-modified carbon paste electrodes," Analytica Chimica Acta, vol. 325, no. 1-2, pp. 33-42, 1996.

[35] D. Pan, J. Chen, S. Yao, W. Tao, and L. Nie, "An amperometric glucose biosensor based on glucose oxidase immobilized in electropolymerized poly (o-aminophenol) and carbon nanotubes composite film on a gold electrode," Analytical Sciences, vol. 21, pp. 367-371, 2005.

[36] Z. Zhang, H. Liu, and J. Deng, "A glucose biosensor based on immobilization of glucose oxidase in electropolymerized $o$-aminophenol film on platinized glassy carbon electrode," Analytical Chemistry, vol. 68, no. 9, pp. 1632-1638, 1996.

[37] J. Li and X. Lin, "Glucose biosensor based on immobilization of glucose oxidase in poly (o-aminophenol) film on polypyrrole-Pt nanocomposite modified glassy carbon electrode," Biosensors and Bioelectronics, vol. 22, no. 12, pp. 2898-2905, 2007.

[38] D. Scolari and R. Tucceri, "Some applications of nonconducting poly(o-aminophenol) films in Bioelectrochemistry and Electrocatalysis," Micro and Nanosystems, vol. 3, pp. 115-130, 2011.

[39] R. I. Tucceri, "Specularity change on a thin gold film surface coated with poly (o-aminophenol) during the polymer redox conversion. The $\mathrm{pH}$ effect on the redox sites distribution at the metal - polymer interface," Journal of Electroanalytical Chemistry, vol. 543, no. 1, pp. 61-71, 2003.

[40] T. Ohsaka, S. Kunimura, and N. Oyama, "Electrode kinetics of poly (o-aminophenol) film prepared by electro-oxidative polymerization of $o$-aminophenol and its electrochromic properties," Electrochimica Acta, vol. 33, no. 5, pp. 639-645, 1988.

[41] H. J. Salavagione, J. Arias-Pardilla, J. M. Pérez et al., "Study of redox mechanism of poly (o-aminophenol) using in situ techniques: evidence of two redox processes," Journal of Electroanalytical Chemistry, vol. 576, no. 1, pp. 139-145, 2005.

[42] R. I. Tucceri, C. Barbero, J. J. Silber, L. Sereno, and D. Posadas, "Spectroelectrochemical study of poly-o-aminophenol," Electrochimica Acta, vol. 42, no. 6, pp. 919-927, 1997.

[43] K. Jackowska, J. Bukowska, and A. Kudelski, "Electro-oxidation of $o$-aminophenol studied by cyclic voltammetry and surface enhanced Raman scattering (SERS)," Journal of Electroanalytical Chemistry, vol. 350, no. 1-2, pp. 177-187, 1993.

[44] Y. Z. Chen, A. Q. Zhang, and Z. W. Tian, "Poly(o-aminophenol) studied by X-ray Photoelectron Spectroscopy," Chemical Journal of Chinese Universities, vol. 12, p. 519, 1991.

[45] C. Barbero, J. Zerbino, L. Sereno, and D. Posadas, "Optical properties of electropolymerized orthoaminophenol," Electrochimica Acta, vol. 32, no. 4, pp. 693-697, 1987.

[46] K. Jackowska, J. Bukowska, and A. Kudelski, "Electro-oxidation of $o$-aminophenol studied by Surface Enhanced Raman Scattering (SERS)," Polish Journal of Chemistry, vol. 68, p. 141, 1994. 

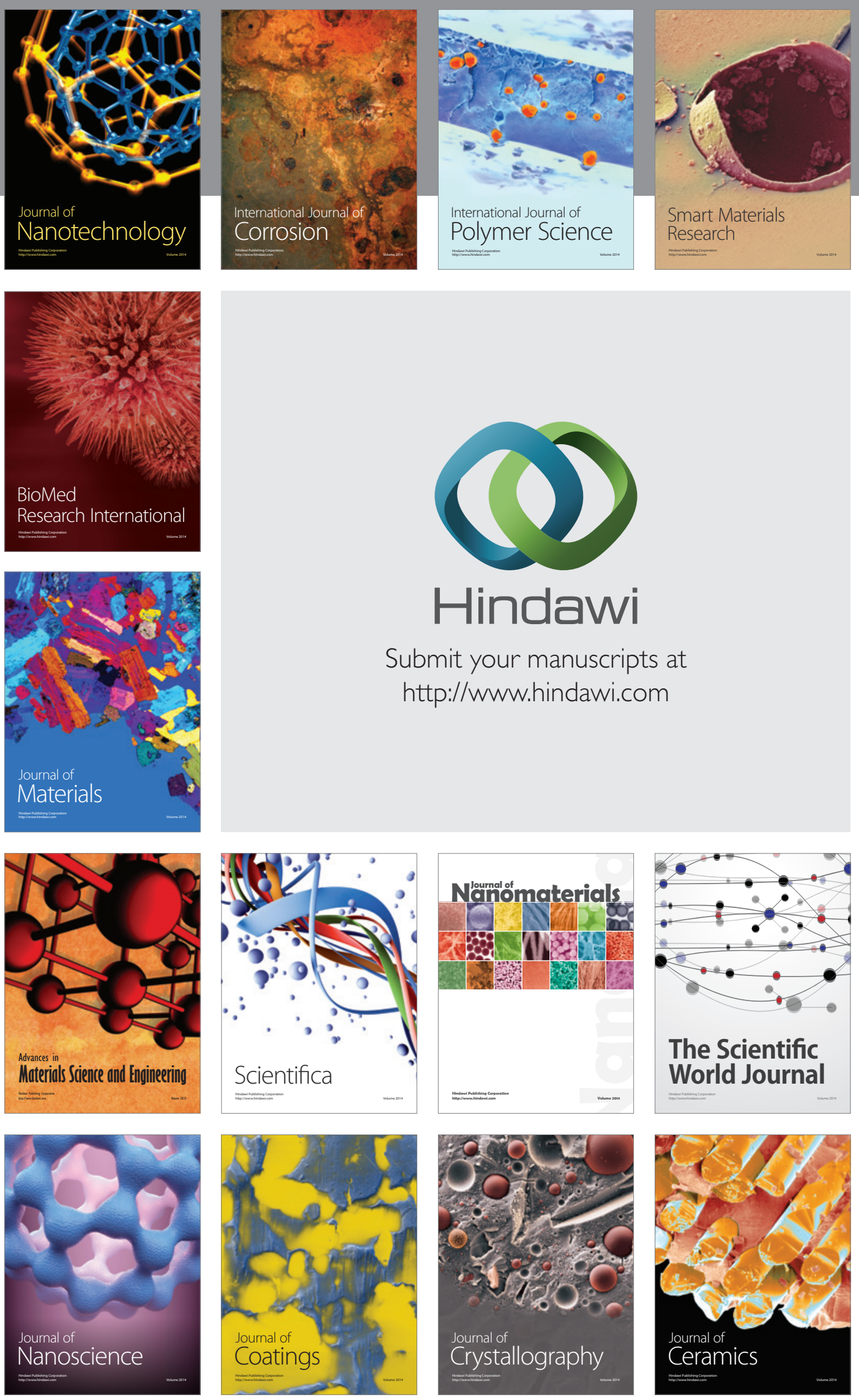

The Scientific World Journal

Submit your manuscripts at

http://www.hindawi.com

\section{World Journal}

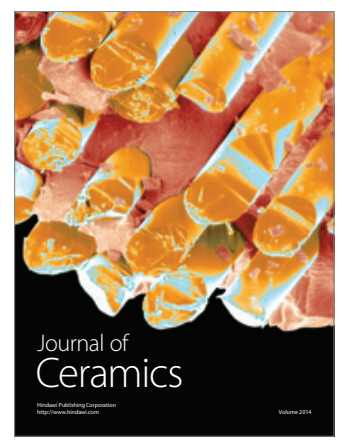

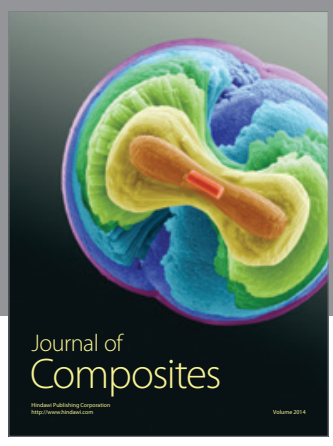
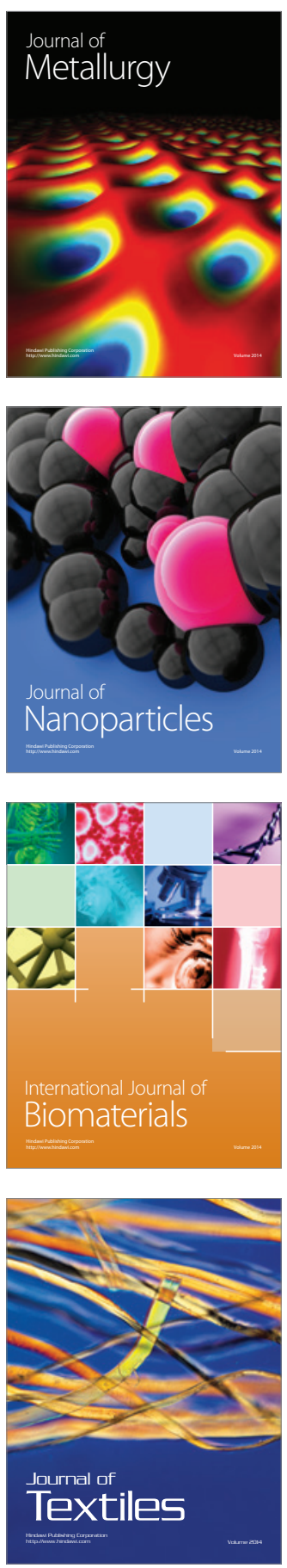